\title{
Diffeomorphism-invariant covariant
}

\author{
Hamiltonians of a
}

\section{pseudo-Riemannian metric and a linear connection}

\author{
Jaime Muñoz Masqué ${ }^{1}$ and María Eugenia Rosado María ${ }^{2}$
}

\author{
${ }^{1}$ Instituto de Física Aplicada, CSIC, C/ Serrano 144, 28006 Madrid, Spain \\ jaime@iec.csic.es \\ ${ }^{2}$ Departamento de Matemática Aplicada, Escuela Técnica Superior de \\ Arquitectura, UPM, Avda. Juan de Herrera 4, 28040 Madrid, Spain \\ eugenia.rosado@upm.es
}

\begin{abstract}
Let $M \rightarrow N$ (resp. $C \rightarrow N$ ) be the fibre bundle of pseudo-Riemannian metrics of a given signature (resp. the bundle of linear connections) on an orientable connected manifold $N$. A geometrically defined class of firstorder Ehresmann connections on the product fibre bundle $M \times{ }_{N} C$ is determined such that, for every connection $\gamma$ belonging to this class and every Diff $N$-invariant Lagrangian density $\Lambda$ on $J^{1}\left(M \times{ }_{N} C\right)$, the corresponding covariant Hamiltonian $\Lambda^{\gamma}$ is also Diff $N$-invariant. The case of Diff $N$-invariant second-order Lagrangian densities on $J^{2} M$ is also studied and the results obtained are then applied to Palatini and Einstein-Hilbert Lagrangians.
\end{abstract}


Mathematics Subject Classification: Primary: 58E30; Secondary: 58A20, 58J70, 83C05.

\section{Introduction}

In Mechanics, the Hamiltonian function attached to a Lagrangian density $\Lambda=L\left(t, q^{i}, \dot{q}^{i}\right) d t$ on $\mathbb{R} \times T Q$ is given by $H=\dot{q}^{i} \partial L / \partial \dot{q}^{i}-L$, but — as it was early observed in [16] - this is not an invariant definition if an arbitrary fibred manifold $t: E \rightarrow \mathbb{R}$ is considered (thus generalizing the notion of an absolute time) instead of the direct product bundle $\mathbb{R} \times Q \rightarrow \mathbb{R}$; e.g., see [7, $23,25]$ for this point of view. In this case, an Ehresmann connection is needed in order to lift the vector field $\partial / \partial t$ from $\mathbb{R}$ to $E$, and the Hamiltonian is then defined by applying the Poincaré-Cartan form attached to $\Lambda$ to the horizontal lift of $\partial / \partial t$.

In the field theory - where no distinguished vector field exists on the base manifold - the need of an Ehresmann connection is even greater, in order to attach a covariant Hamiltonian to each Lagrangian density; e.g., see $[23,24,4.1]$, and the definitions below.

Let $p: E \rightarrow N$ be an arbitrary fibred manifold over a connected manifold $N, n=\operatorname{dim} N, \operatorname{dim} E=m+n$, oriented by $v_{n}=d x^{1} \wedge \cdots \wedge d x^{n}$. Throughout this paper, Latin (resp. Greek) indices run from 1 to $n$ (resp. $m$ ). An Ehresmann connection on a fibred manifold $p: E \rightarrow N$ is a differential 1-form $\gamma$ on $E$ taking values in the vertical sub-bundle $V(p)$ such that $\gamma(X)=X$ for every $X \in V(p)$ (e.g., see $[23,24,32,34]$ ). Once an Ehresmann connection $\gamma$ is given, a decomposition of vector bundles holds $T(E)=V(p) \oplus \operatorname{ker} \gamma$, where $\operatorname{ker} \gamma$ is called the horizontal sub-bundle determined by $\gamma$. In a fibred coordinate system $\left(x^{j}, y^{\alpha}\right)$ for $p$, an Ehresmann connection can be written as

$$
\gamma=\left(d y^{\alpha}+\gamma_{j}^{\alpha} d x^{j}\right) \otimes \frac{\partial}{\partial y^{\alpha}}, \quad \gamma_{j}^{\alpha} \in C^{\infty}(E) .
$$

According to [24], the covariant Hamiltonian $\Lambda^{\gamma}$ associated to a Lagrangian density on $J^{1} E, \Lambda=L v_{n}, L \in C^{\infty}\left(J^{1} E\right)$, with respect to $\gamma$ is the Lagrangian density defined by,

$$
\Lambda^{\gamma}=\left(\left(p_{0}^{1}\right)^{*} \gamma-\theta\right) \wedge \omega_{\Lambda}-\Lambda
$$

where, $p_{0}^{1}: J^{1} E \rightarrow J^{0} E=E$ is the projection mapping, $\theta=\theta^{\alpha} \otimes \partial / \partial y^{\alpha}$, $\theta^{\alpha}=d y^{\alpha}-y_{i}^{\alpha} d x^{i}$ is the $V(p)$-valued 1-form on $J^{1} E$ associated with the contact structure, written on a fibred coordinate system $\left(x^{i}, y^{\alpha}\right)$, and $\omega_{\Lambda}$ 
is the Legendre form attached to $\Lambda$, i.e., the $V^{*}(p)$-valued $p^{1}$-horizontal $(n-1)$-form on $J^{1} E$ given by

$$
\omega_{\Lambda}=(-1)^{i-1} \frac{\partial L}{\partial y_{i}^{\alpha}} i_{\partial / \partial x^{i}} v_{n} \otimes d y^{\alpha}
$$

where $\left(x^{i}, y^{\alpha} ; y_{i}^{\alpha}\right)$ is the coordinate system induced from $\left(x^{i}, y^{\alpha}\right)$ on the 1-jet bundle and $p^{1}: J^{1} E \rightarrow N$ is the projection on the base manifold. Locally,

$$
\Lambda^{\gamma}=\left(\left(\gamma_{i}^{\alpha}+y_{i}^{\alpha}\right) \frac{\partial L}{\partial y_{i}^{\alpha}}-L\right) d x^{1} \wedge \cdots \wedge d x^{n}
$$

From (1.1) we obtain the following decomposition of the Poincaré-Cartan form attached to $\Lambda$ (e.g., see $[17,23,27]): \Theta_{\Lambda}=\theta \wedge \omega_{\Lambda}+\Lambda=\left(p_{0}^{1}\right)^{*} \gamma \wedge$ $\omega_{\Lambda}-\Lambda^{\gamma}$.

A diffeomorphism $\Phi: E \rightarrow E$ is said to be an automorphism of $p$ if there exists $\phi \in \operatorname{Diff} N$ such that $p \circ \Phi=\phi \circ p$. The set of such automorphisms is denoted by $\operatorname{Aut}(p)$ and its Lie algebra is identified to the space $\operatorname{aut}(p) \subset$ $\mathfrak{X}(E)$ of $p$-projectable vector fields on $E$. Given a subgroup $\mathcal{G} \subseteq \operatorname{Aut}(p)$, a Lagrangian density $\Lambda$ is said to be $\mathcal{G}$-invariant if $\left(\Phi^{(1)}\right)^{*} \Lambda=\Lambda$ for every $\Phi \in$ $\mathcal{G}$, where $\Phi^{(1)}: J^{1} E \rightarrow J^{1} E$ denotes the 1-jet prolongation of $\Phi$. Infinitesimally, the $\mathcal{G}$-invariance equation can be reformulated as $L_{X^{(1)}} \Lambda=0$ for every $X \in \operatorname{Lie}(\mathcal{G}), X^{(1)}$ denoting the 1-jet prolongation of the vector field $X$.

When a group $\mathcal{G}$ of transformations of $E$ is given, a natural question arises:

- Determine a class - as small as possible - of Ehresmann connections $\gamma$ such that $\Lambda^{\gamma}$ is $\mathcal{G}$-invariant for every $\mathcal{G}$-invariant Lagrangian density $\Lambda$.

Below we tackle this question in the framework of General Relativity, i.e., the group $\mathcal{G}$ is the group of all diffeomorphisms of the ground manifold $N$ acting in a natural way either on the bundle of pseudo-Riemannian metrics $p_{M}: M=M(N) \rightarrow N$ of a given signature $\left(n^{+}, n^{-}\right), n^{+}+n^{-}=n$, or on the product bundle $p: M \times_{N} C \rightarrow N$, where $p_{C}: C=C(N) \rightarrow N$ is the bundle of linear connections on $N$. Namely, we solve the following two problems:

(P): Determine a class — as small as possible - of Ehresmann connections $\gamma$ such that for every Diff $N$-invariant first-order Lagrangian density $\Lambda$ on the bundle $J^{1}\left(M \times{ }_{N} C\right)$, the corresponding covariant Hamiltonian $\Lambda^{\gamma}$ is also Diff $N$-invariant. 
Similarly to the problem $(\mathbf{P})$, we formulate the corresponding problem on $J^{2} M$ as follows:

(P2): Determine a class of second-order Ehresmann connections $\gamma^{2}$ on $M$ such that for every Diff $N$-invariant second-order Lagrangian density $\Lambda$ on the bundle $J^{2} M$, the corresponding covariant Hamiltonian $\Lambda^{\gamma^{2}}$ — defined in (4.9) — is also Diff $N$-invariant.

Essentially, a class of first-order Ehresmann connections on the bundle $M \times{ }_{N} C$ is obtained, defined by the conditions $\left(C_{M}\right)$ and $\left(C_{C}\right)$ below (see Propositions 3.4 and 3.5), solving the problem (P). This class of connections also helps to solve (P2) by means of a natural isomorphism between $J^{1} M$ and $M \times{ }_{N} C^{\mathrm{sym}}$, where $C^{\mathrm{sym}}$ denotes the sub-bundle of symmetric connections on $N$ (cf. Theorem 4.1). Finally, this approach is applied to Palatini and Einstein-Hilbert Lagrangians [3,4], obtaining results compatible with their usual Hamiltonian formalisms.

\section{Invariance under diffeomorphisms}

\subsection{Preliminaries}

\subsubsection{Jet-bundle notations}

Let $p^{k}: J^{k} E \rightarrow N$ be the $k$-jet bundle of local sections of an arbitrary fibred manifold $p: E \rightarrow N$, with projections $p_{l}^{k}: J^{k} E \rightarrow J^{l} E, p_{l}^{k}\left(j_{x}^{k} s\right)=j_{x}^{l} s$, for $k \geq l, j_{x}^{k} s$ denoting the $k$-jet at $x$ of a section $s$ of $p$ defined on a neighbourhood of $x \in N$.

A fibred coordinate system $\left(x^{i}, y^{\alpha}\right)$ on $V$ induces a coordinate system $\left(x^{i}, y_{I}^{\alpha}\right), I=\left(i_{1}, \ldots, i_{n}\right) \in \mathbb{N}^{n}, 0 \leq|I|=i_{1}+\cdots+i_{n} \leq r$, on $\left(p_{0}^{r}\right)^{-1}(V)=$ $J^{r} V$ as follows: $y_{I}^{\alpha}\left(j_{x}^{r} s\right)=\left(\partial^{|I|}\left(y^{\alpha} \circ s\right) / \partial x^{I}\right)(x)$, with $y_{0}^{\alpha}=y^{\alpha}$.

Every morphism $\Phi: E \rightarrow E^{\prime}$ whose associated map $\phi: N \rightarrow N^{\prime}$ is a diffeomorphism, induces a map

$$
\begin{array}{r}
\Phi^{(r)}: J^{r} E \rightarrow J^{r} E^{\prime}, \\
\Phi^{(r)}\left(j_{x}^{r} s\right)=j_{\phi(x)}^{r}\left(\Phi \circ s \circ \phi^{-1}\right) .
\end{array}
$$

If $\Phi_{t}$ is the flow of a vector field $X \in \operatorname{aut}(p)$, then $\Phi_{t}^{(r)}$ is the flow of a vector field $X^{(r)} \in \mathfrak{X}\left(J^{r} E\right)$, called the infinitesimal contact transformation of order 
$r$ associated to the vector field $X$. The mapping

$$
\operatorname{aut}(p) \ni X \mapsto X^{(r)} \in \mathfrak{X}\left(J^{r} E\right)
$$

is an injection of Lie algebras, namely, one has

$$
\begin{aligned}
& (\lambda X+\mu Y)^{(r)}=\lambda X^{(r)}+\mu Y^{(r)}, \\
& {[X, Y]^{(r)}=\left[X^{(r)}, Y^{(r)}\right],} \\
& \forall \lambda, \mu \in \mathbb{R}, \forall X, Y \in \operatorname{aut}(p) .
\end{aligned}
$$

In particular, for $r=1$,

$$
\begin{aligned}
X & =u^{i} \frac{\partial}{\partial x^{i}}+v^{\alpha} \frac{\partial}{\partial y^{\alpha}}, \quad u^{i} \in C^{\infty}(N), v^{\alpha} \in C^{\infty}(E), \\
X^{(1)} & =u^{i} \frac{\partial}{\partial x^{i}}+v^{\alpha} \frac{\partial}{\partial y^{\alpha}}+v_{i}^{\alpha} \frac{\partial}{\partial y_{i}^{\alpha}}, \quad v_{i}^{\alpha}=\frac{\partial v^{\alpha}}{\partial x^{i}}+y_{i}^{\beta} \frac{\partial v^{\alpha}}{\partial y^{\beta}}-y_{k}^{\alpha} \frac{\partial u^{k}}{\partial x^{i}} .
\end{aligned}
$$

\subsubsection{Coordinates on $M(N), F(N)$, and $C(N)$}

Every coordinate system $\left(x^{i}\right)$ on an open domain $U \subseteq N$ induces the following coordinate systems:

(1) $\left(x^{i}, y_{j k}\right)$ on $\left(p_{M}\right)^{-1}(U)$, where $p_{M}: M \rightarrow N$ is the bundle of metrics of a given signature, and the functions $y_{j k}=y_{k j}$ are defined by,

$$
g_{x}=\sum_{i \leq j} y_{i j}\left(g_{x}\right)\left(d x^{i}\right)_{x} \otimes\left(d x^{j}\right)_{x}, \forall g_{x} \in\left(p_{M}\right)^{-1}(U) .
$$

(2) $\left(x^{i}, x_{j}^{i}\right)$ on $\left(p_{F}\right)^{-1}(U)$, where $p_{F}: F(N) \rightarrow N$ is the bundle of linear frames on $N$, and the functions $x_{j}^{i}$ are defined by,

$u=\left(\left(\partial / \partial x^{1}\right)_{x}, \ldots,\left(\partial / \partial x^{n}\right)_{x}\right) \cdot\left(x_{j}^{i}(u)\right), \quad x=p_{F}(u), \forall u \in\left(p_{F}\right)^{-1}(U)$,

or equivalently,

$$
u=\left(X_{1}, \ldots, X_{n}\right) \in F_{x}(N), X_{j}=x_{j}^{i}(u)\left(\frac{\partial}{\partial x^{i}}\right)_{x}, \quad 1 \leq j \leq n .
$$

(3) $\left(x^{i}, A_{k l}^{j}\right)$ on $\left(p_{C}\right)^{-1}(U)$, where $p_{C}: C \rightarrow N$ is the bundle of linear connections on $N$, and the functions $A_{k l}^{j}$ are defined as follows. We first 
recall some basic facts. Connections on $F(N)$ (i.e., linear connections of $N$ ) are the splittings of the Atiyah sequence (cf. [2]),

$$
0 \rightarrow \operatorname{ad} F(N) \rightarrow T_{G l(n, \mathbb{R})} F(N) \stackrel{\left(p_{F}\right)_{*}}{\longrightarrow} T N \rightarrow 0,
$$

where

(a) $\operatorname{ad} F(N)=T^{*} N \otimes T N$ is the adjoint bundle;

(b) $T_{G l(n, \mathbb{R})}(F(N))=T(F(N)) / G l(n, \mathbb{R})$; and

(c) $\operatorname{gau} F(N)=\Gamma(N, \operatorname{ad} F(N))$ is the gauge algebra of $F(N)$.

We think of gau $F(N)$ as the 'Lie algebra' of the gauge group $\operatorname{Gau} F(N)$. Moreover, $p_{C}: C \rightarrow N$ is an affine bundle modelled over the vector bundle $\otimes^{2} T^{*} N \otimes T N$. The section of $p_{C}$ induced tautologically by the linear connection $\Gamma$ is denoted by $s_{\Gamma}: N \rightarrow C$. Every $B \in \mathfrak{g l}(n, \mathbb{R})$ defines a one-parameter group $\varphi_{t}^{B}: U \times G l(n, \mathbb{R}) \rightarrow U \times G l(n, \mathbb{R})$ of gauge transformations by setting (cf. [5]), $\varphi_{t}^{B}(x, \Lambda)=(x, \exp (t B) \cdot \Lambda)$. Let us denote by $\bar{B} \in \operatorname{gau}\left(p_{F}\right)^{-1}(U)$ the corresponding infinitesimal generator. If $\left(E_{j}^{i}\right)$ is the standard basis of $\mathfrak{g l}(n, \mathbb{R})$, then $\bar{E}_{j}^{i}=\sum_{h=1}^{n}$ $x_{h}^{j} \partial / \partial x_{h}^{i}$, for $i, j=1, \ldots, n$, is a basis of $\operatorname{gau}\left(p_{F}\right)^{-1}(U)$. Let $\tilde{E}_{j}^{i}=$ $\bar{E}_{j}^{i} \bmod G$ be the class of $\bar{E}_{j}^{i}$ on $\operatorname{ad} F(N)$. Unique smooth functions $A_{j k}^{i}$ on $\left(p_{C}\right)^{-1}(U)$ exist such that,

$$
\begin{aligned}
s_{\Gamma}\left(\frac{\partial}{\partial x^{j}}\right) & =\frac{\partial}{\partial x^{j}}-\left(A_{j k}^{i} \circ \Gamma\right) \tilde{E}_{k}^{i} \\
& =\frac{\partial}{\partial x^{j}}-\left(A_{j k}^{i} \circ \Gamma\right) x_{h}^{k} \frac{\partial}{\partial x_{h}^{i}},
\end{aligned}
$$

for every $s_{\Gamma}$ and $A_{j k}^{i}\left(\Gamma_{x}\right)=\Gamma_{j k}^{i}(x)$, where $\Gamma_{j k}^{i}$ are the Christoffel symbols of the linear connection $\Gamma$ in the coordinate system $\left(x^{i}\right)$, see $[20$, III, Proposition 7.4].

\section{$2.2 \quad$ Natural lifts}

Let $f_{M}: M \rightarrow M$, cf. [30] (resp. $\tilde{f}: F(N) \rightarrow F(N)$, cf. [20, p. 226]) be the natural lift of $f \in \operatorname{Diff} N$ to the bundle of metrics (resp. linear frame bundle); namely $f_{M}\left(g_{x}\right)=\left(f^{-1}\right)^{*} g_{x}\left(\operatorname{resp} . \tilde{f}\left(X_{1}, \ldots, X_{n}\right)=\left(f_{*} X_{1}, \ldots, f_{*} X_{n}\right)\right.$, where $\left.\left(X_{1}, \ldots, X_{n}\right) \in F_{x}(N)\right)$; hence $p_{M} \circ f_{M}=f \circ p_{M}$ (resp. $p_{F} \circ \tilde{f}=f \circ$ $p_{F}$ ), and $f_{M}: M \rightarrow M$ (resp. $\tilde{f}: F(N) \rightarrow F(N)$ ) have a natural extension to jet bundles $f_{M}^{(r)}: J^{r}(M) \rightarrow J^{r}(M)$ (resp. $\left.\tilde{f}^{(r)}: J^{r}(F N) \rightarrow J^{r}(F N)\right)$ as 
defined in the formula (2.1), i.e.,

$$
f_{M}^{(r)}\left(j_{x}^{r} g\right)=j_{f(x)}^{r}\left(f_{M} \circ g \circ f^{-1}\right) \quad\left(\operatorname{resp} . \tilde{f}^{(r)}\left(j_{x}^{r} s\right)=j_{f(x)}^{r}\left(\tilde{f} \circ s \circ f^{-1}\right)\right) .
$$

As $\tilde{f}$ is an automorphism of the principal $G l(n, \mathbb{R})$-bundle $F(N)$, it acts on linear connections by pulling back connection forms, i.e., $\Gamma^{\prime}=\tilde{f}(\Gamma)$ where $\omega_{\Gamma^{\prime}}=\left(\tilde{f}^{-1}\right)^{*} \omega_{\Gamma}$ (see [20, II, Proposition 6.2-(b)], [5, 3.3]). Hence, there exists a unique diffeomorphism $\tilde{f}_{C}: C \rightarrow C$ such that,

(1) $p_{C} \circ \tilde{f}_{C}=f \circ p_{C}$, and

(2) $\tilde{f}_{C} \circ s_{\Gamma}=s_{\tilde{f}(\Gamma)}$ for every linear connection $\Gamma$.

If $f_{t}$ is the flow of a vector field $X \in \mathfrak{X}(N)$, then the infinitesimal generator of $\left(f_{t}\right)_{M}\left(\operatorname{resp} . \tilde{f}_{t}\right.$, resp. $\left.\left(\tilde{f}_{t}\right)_{C}\right)$ in $\operatorname{Diff} M($ resp. Diff $F(N)$, resp. Diff $C)$ is denoted by $X_{M}$ (resp. $\tilde{X}$, resp. $\tilde{X}_{C}$ ) and the following Lie-algebra homomorphisms are obtained:

$$
\begin{cases}\mathfrak{X}(N) \rightarrow \mathfrak{X}(M), & X \mapsto X_{M}, \\ \mathfrak{X}(N) \rightarrow \mathfrak{X}(F(N)), & X \mapsto \tilde{X}, \\ \mathfrak{X}(N) \rightarrow \mathfrak{X}(C), & X \mapsto \tilde{X}_{C} .\end{cases}
$$

If $X=u^{i} \partial / \partial x^{i} \in \mathfrak{X}(N)$ is the local expression for $X$, then

(1) From [30, equations (2) to (4)] we know that the natural lift of $X$ to $M$ is given by,

$$
X_{M}=u^{i} \frac{\partial}{\partial x^{i}}-\sum_{i \leq j}\left(\frac{\partial u^{h}}{\partial x^{i}} y_{h j}+\frac{\partial u^{h}}{\partial x^{j}} y_{i h}\right) \frac{\partial}{\partial y_{i j}} \in \mathfrak{X}(M) .
$$

and its 1-jet prolongation,

$$
\begin{aligned}
X_{M}^{(1)}= & u^{i} \frac{\partial}{\partial x^{i}}-\sum_{i \leq j}\left(\frac{\partial u^{h}}{\partial x^{i}} y_{h j}+\frac{\partial u^{h}}{\partial x^{j}} y_{h i}\right) \frac{\partial}{\partial y_{i j}} \\
& -\sum_{i \leq j}\left(\frac{\partial^{2} u^{h}}{\partial x^{i} \partial x^{k}} y_{h j}+\frac{\partial^{2} u^{h}}{\partial x^{j} \partial x^{k}} y_{h i}+\frac{\partial u^{h}}{\partial x^{i}} y_{h j, k}+\frac{\partial u^{h}}{\partial x^{j}} y_{h i, k}+\frac{\partial u^{h}}{\partial x^{k}} y_{i j, h}\right) \\
& \times \frac{\partial}{\partial y_{i j, k}} .
\end{aligned}
$$


(2) From [10, Proposition 3] (also see [20, VI, Proposition 21.1]) we know that the natural lift of $X$ to $F(N)$ is given by

$$
\tilde{X}=u^{i} \frac{\partial}{\partial x^{i}}+\frac{\partial u^{i}}{\partial x^{l}} x_{j}^{l} \frac{\partial}{\partial x_{j}^{i}},
$$

and its 1-jet prolongation

$$
\begin{aligned}
\tilde{X}^{(1)} & =u^{i} \frac{\partial}{\partial x^{i}}+\frac{\partial u^{i}}{\partial x^{l}} x_{j}^{l} \frac{\partial}{\partial x_{j}^{i}}+v_{j k}^{i} \frac{\partial}{\partial x_{j, k}^{i}}, \\
v_{j k}^{i} & =\frac{\partial u^{i}}{\partial x^{l}} x_{j, k}^{l}-\frac{\partial u^{l}}{\partial x^{k}} x_{j, l}^{i}+\frac{\partial^{2} u^{i}}{\partial x^{k} \partial x^{l}} x_{j}^{l} .
\end{aligned}
$$

(3) Finally,

$$
\begin{aligned}
\tilde{X}_{C}= & u^{i} \frac{\partial}{\partial x^{i}}-\left(\frac{\partial^{2} u^{i}}{\partial x^{j} \partial x^{k}}-\frac{\partial u^{i}}{\partial x^{l}} A_{j k}^{l}+\frac{\partial u^{l}}{\partial x^{k}} A_{j l}^{i}+\frac{\partial u^{l}}{\partial x^{j}} A_{l k}^{i}\right) \frac{\partial}{\partial A_{j k}^{i}}, \\
\tilde{X}_{C}^{(1)}= & u^{i} \frac{\partial}{\partial x^{i}}+w_{j k}^{i} \frac{\partial}{\partial A_{j k}^{i}}+w_{j k h}^{i} \frac{\partial}{\partial A_{j k, h}^{i}}, \\
w_{j k}^{i}= & -\frac{\partial^{2} u^{i}}{\partial x^{j} \partial x^{k}}+\frac{\partial u^{i}}{\partial x^{l}} A_{j k}^{l}-\frac{\partial u^{l}}{\partial x^{k}} A_{j l}^{i}-\frac{\partial u^{l}}{\partial x^{j}} A_{l k}^{i}, \\
w_{j k h}^{i}= & -\frac{\partial^{3} u^{i}}{\partial x^{h} \partial x^{j} \partial x^{k}}+\frac{\partial^{2} u^{i}}{\partial x^{h} \partial x^{l}} A_{j k}^{l}-\frac{\partial^{2} u^{l}}{\partial x^{h} \partial x^{k}} A_{j l}^{i}-\frac{\partial^{2} u^{l}}{\partial x^{h} \partial x^{j}} A_{l k}^{i} \\
& +\frac{\partial u^{i}}{\partial x^{l}} A_{j k, h}^{l}-\frac{\partial u^{l}}{\partial x^{k}} A_{j l, h}^{i}-\frac{\partial u^{l}}{\partial x^{j}} A_{l k, h}^{i}-\frac{\partial u^{l}}{\partial x^{h}} A_{j k, l}^{i} .
\end{aligned}
$$

Let $p: M \times{ }_{N} C \rightarrow N$ be the natural projection.

We denote by $\bar{f}=\left(f_{M}, \tilde{f}_{C}\right)\left(\right.$ resp. $\left.\bar{X}=\left(X_{M}, \tilde{X}_{C}\right) \in \mathfrak{X}\left(M \times_{N} C\right)\right)$ the natural lift of $f$ (resp. $X)$ to $M \times_{N} C$. The prolongation to the bundle $J^{1}\left(M \times_{N}\right.$ $C)$ of $\bar{X}$ is as follows:

$$
\begin{aligned}
\bar{X}^{(1)}= & \left(X_{M}^{(1)}, \tilde{X}_{C}^{(1)}\right)=u^{i} \frac{\partial}{\partial x^{i}} \\
& +\sum_{i \leq j} v_{i j} \frac{\partial}{\partial y_{i j}}+\sum_{i \leq j} v_{i j k} \frac{\partial}{\partial y_{i j, k}}+w_{j k}^{i} \frac{\partial}{\partial A_{j k}^{i}}+w_{j k h}^{i} \frac{\partial}{\partial A_{j k, h}^{i}},
\end{aligned}
$$

where

$$
\begin{aligned}
v_{i j} & =-\frac{\partial u^{h}}{\partial x^{i}} y_{h j}-\frac{\partial u^{h}}{\partial x^{j}} y_{h i}, \\
v_{i j k} & =-\frac{\partial^{2} u^{h}}{\partial x^{i} \partial x^{k}} y_{h j}-\frac{\partial^{2} u^{h}}{\partial x^{j} \partial x^{k}} y_{h i}-\frac{\partial u^{h}}{\partial x^{i}} y_{h j, k}-\frac{\partial u^{h}}{\partial x^{j}} y_{h i, k}-\frac{\partial u^{h}}{\partial x^{k}} y_{i j, h},
\end{aligned}
$$

and $w_{j k}^{i}, w_{j k h}^{i}$ are given in the formulas (2.5) and (2.6), respectively. 


\subsection{Diff $N$ - and $\mathfrak{X}(N)$-invariance}

A differential form $\omega_{r} \in \Omega^{r}\left(J^{1}\left(M \times_{N} C\right)\right), r \in \mathbb{N}$, is said to be Diff $N$ invariant - or invariant under diffeomorphisms - (resp. $\mathfrak{X}(N)$-invariant) if the following equation holds: $\left(\bar{f}^{(1)}\right)^{*} \omega_{r}=\omega_{r}, \forall f \in \operatorname{Diff} N$ (resp. $L_{\bar{X}^{(1)}} \omega_{r}=0$, $\forall X \in \mathfrak{X}(N))$. Obviously, "Diff $N$-invariance" implies " $\mathfrak{X}(N)$-invariance" and the converse is almost true (see $[14,28]$ ). Because of this, below we consider $\mathfrak{X}(N)$-invariance only.

A linear frame $\left(X_{1}, \ldots, X_{n}\right)$ at $x$ is said to be orthonormal with respect to $g_{x} \in M_{x}(N)$ (or simply $g_{x}$-orthonormal) if $g_{x}\left(X_{i}, X_{j}\right)=0$ for $1 \leq i<j \leq n$, $g\left(X_{i}, X_{i}\right)=1$ for $1 \leq i \leq n^{+}, g\left(X_{i}, X_{i}\right)=-1$ for $n^{+}+1 \leq i \leq n$.

As $N$ is an oriented manifold, there exists a unique $p$-horizontal $n$-form $\mathbf{v}$ on $M \times{ }_{N} C$ such that, $\mathbf{v}_{\left(g_{x}, \Gamma_{x}\right)}\left(X_{1}, \ldots, X_{n}\right)=1$, for every $g_{x}$-orthonormal basis $\left(X_{1}, \ldots, X_{n}\right)$ belonging to the orientation of $N$. Locally $\mathbf{v}=\rho v_{n}$, where $\rho=\sqrt{(-1)^{n^{-}} \operatorname{det}\left(y_{i j}\right)}$ and $v_{n}=d x^{1} \wedge \cdots \wedge d x^{n}$. As proved in [30, Proposition 7], the form $\mathbf{v}$ is Diff $N$-invariant and hence $\mathfrak{X}(N)$-invariant. A Lagrangian density $\Lambda$ on $J^{1}\left(M \times{ }_{N} C\right)$ can be globally written as $\Lambda=\mathcal{L} \mathbf{v}$ for a unique function $\mathcal{L} \in C^{\infty}\left(J^{1}\left(M \times{ }_{N} C\right)\right)$ and $\Lambda$ is $\mathfrak{X}(N)$-invariant if and only if the function $\mathcal{L}$ is. Therefore, the invariance of Lagrangian densities is reduced to that of scalar functions.

Proposition 2.1. A function $\mathcal{L} \in C^{\infty}\left(J^{1}\left(M \times_{N} C\right)\right)$ is $\mathfrak{X}(N)$-invariant if and only if the following system of partial differential equations hold:

$$
\begin{cases}0=X^{i}(\mathcal{L}), & \forall i, \\ 0=X_{h}^{i}(\mathcal{L}), & \forall h, i, \\ 0=X_{h}^{i k}(\mathcal{L}), & \forall h, i \leq k, \\ 0=X_{i}^{j k h}(\mathcal{L}), & \forall i, j \leq k \leq h,\end{cases}
$$

where

$$
\begin{aligned}
X^{i}= & \frac{\partial}{\partial x^{i}}, \quad \forall i \\
X_{h}^{i}= & -y_{h i} \frac{\partial}{\partial y_{i i}}-y_{h j} \frac{\partial}{\partial y_{i j}}-y_{i h, k} \frac{\partial}{\partial y_{i i, k}}-y_{h j, k} \frac{\partial}{\partial y_{i j, k}}-\sum_{s \leq j} y_{s j, h} \frac{\partial}{\partial y_{s j, i}} \\
& +A_{j k}^{i} \frac{\partial}{\partial A_{j k}^{h}}-A_{j h}^{r} \frac{\partial}{\partial A_{j i}^{r}}-A_{h k}^{r} \frac{\partial}{\partial A_{i k}^{r}} \\
& +A_{j k, s}^{i} \frac{\partial}{\partial A_{j k, s}^{h}}-A_{j h, r}^{s} \frac{\partial}{\partial A_{j i, r}^{s}}-A_{h k, r}^{s} \frac{\partial}{\partial A_{i k, r}^{s}}-A_{j k, h}^{r} \frac{\partial}{\partial A_{j k, i}^{r}}, \forall h, i,
\end{aligned}
$$




$$
\begin{aligned}
X_{h}^{i k}= & -y_{i h} \frac{\partial}{\partial y_{i i, k}}-y_{k h} \frac{\partial}{\partial y_{k k, i}}-y_{h j} \frac{\partial}{\partial y_{i j, k}}-y_{h j} \frac{\partial}{\partial y_{k j, i}}-\frac{\partial}{\partial A_{i k}^{h}}-\frac{\partial}{\partial A_{k i}^{h}} \\
& +A_{j s}^{k} \frac{\partial}{\partial A_{j s, i}^{h}}-A_{j h}^{s} \frac{\partial}{\partial A_{j k, i}^{s}}-A_{h r}^{s} \frac{\partial}{\partial A_{k r, i}^{s}} \\
& +A_{j s}^{i} \frac{\partial}{\partial A_{j s, k}^{h}}-A_{j h}^{s} \frac{\partial}{\partial A_{j i, k}^{s}}-A_{h r}^{s} \frac{\partial}{\partial A_{i r, k}^{s}}, \quad \forall h, i \leq k, \\
X_{i}^{j k h}= & \frac{\partial}{\partial A_{j k, h}^{i}}+\frac{\partial}{\partial A_{j h, k}^{i}}+\frac{\partial}{\partial A_{h k, j}^{i}}+\frac{\partial}{\partial A_{h j, k}^{i}}+\frac{\partial}{\partial A_{k j, h}^{i}}+\frac{\partial}{\partial A_{k h, j}^{i}}, \\
& \forall i, h \leq j \leq k .
\end{aligned}
$$

Moreover, the vector fields $X^{i}, X_{h}^{i}, X_{h}^{i k}, X_{i}^{j k h}$ are linearly independent and they span an involutive distribution on $J^{1}\left(M \times{ }_{N} C\right)$ of rank $n\left(\begin{array}{c}n+3 \\ 3\end{array}\right)$. Hence, the number of functionally invariant Lagrangians on $J^{1}\left(M \times{ }_{N} C\right)$ is

$$
\frac{1}{6}\left(5 n^{4}+3 n^{3}-5 n^{2}+3 n\right)
$$

Proof. According to the formula $(2.7), \mathcal{L}$ is invariant if and only if,

$$
\begin{aligned}
u^{i} \frac{\partial \mathcal{L}}{\partial x^{i}} & +\sum_{i \leq j} v_{i j} \frac{\partial \mathcal{L}}{\partial y_{i j}}+\sum_{i \leq j} v_{i j k} \frac{\partial \mathcal{L}}{\partial y_{i j, k}}+w_{j k}^{i} \frac{\partial \mathcal{L}}{\partial A_{j k}^{i}}+w_{j k h}^{i} \frac{\partial \mathcal{L}}{\partial A_{j k, h}^{i}}=0, \\
\forall u^{i} & \in C^{\infty}(N),
\end{aligned}
$$

and expanding on this equation by using the formulas (2.8), (2.9), (2.5) and (2.6), we obtain

$$
\begin{aligned}
0= & u^{i} \frac{\partial \mathcal{L}}{\partial x^{i}} \\
& +\frac{\partial u^{h}}{\partial x^{i}}\left(-y_{h i} \frac{\partial \mathcal{L}}{\partial y_{i i}}-y_{h j} \frac{\partial \mathcal{L}}{\partial y_{i j}}-y_{i h, k} \frac{\partial \mathcal{L}}{\partial y_{i i, k}}-y_{h j, k} \frac{\partial \mathcal{L}}{\partial y_{i j, k}}\right. \\
& -\sum_{s \leq j} y_{s j, h} \frac{\partial \mathcal{L}}{\partial y_{s j, i}}+A_{j k}^{i} \frac{\partial \mathcal{L}}{\partial A_{j k}^{h}}-A_{j h}^{r} \frac{\partial \mathcal{L}}{\partial A_{j i}^{r}}-A_{h k}^{r} \frac{\partial \mathcal{L}}{\partial A_{i k}^{r}} \\
& \left.+A_{j k, s}^{i} \frac{\partial \mathcal{L}}{\partial A_{j k, s}^{h}}-A_{j h, r}^{s} \frac{\partial \mathcal{L}}{\partial A_{j i, r}^{s}}-A_{h k, r}^{s} \frac{\partial \mathcal{L}}{\partial A_{i k, r}^{s}}-A_{j k, h}^{r} \frac{\partial \mathcal{L}}{\partial A_{j k, i}^{r}}\right)
\end{aligned}
$$




$$
\begin{aligned}
& +\frac{\partial^{2} u^{h}}{\partial x^{i} \partial x^{k}}\left(-y_{i h} \frac{\partial \mathcal{L}}{\partial y_{i i, k}}-y_{h j} \frac{\partial \mathcal{L}}{\partial y_{i j, k}}-\frac{\partial \mathcal{L}}{\partial A_{i k}^{h}}\right. \\
& \left.+A_{j s}^{k} \frac{\partial \mathcal{L}}{\partial A_{j s, i}^{h}}-A_{j h}^{s} \frac{\partial \mathcal{L}}{\partial A_{j k, i}^{s}}-A_{h r}^{r} \frac{\partial \mathcal{L}}{\partial A_{k r, i}^{r}}\right) \\
& -\frac{\partial^{3} u^{i}}{\partial x^{h} \partial x^{k} \partial x^{j}} \frac{\partial \mathcal{L}}{\partial A_{j k, h}^{i}} .
\end{aligned}
$$

This equation is equivalent to the system of the statement as the values for $u^{h}, \partial u^{h} / \partial x^{i}, \partial^{2} u^{h} / \partial x^{i} \partial x^{j}(i \leq j)$, and $\partial^{3} u^{h} / \partial x^{i} \partial x^{j} \partial x^{k}(i \leq j \leq k)$ at a point $x \in N$ can be taken arbitrarily. Moreover, assume a linear combination holds

$$
\begin{aligned}
& \lambda_{a} X^{a}+\lambda_{b}^{a} X_{a}^{b}+\sum_{b \leq c} \lambda_{b c}^{a} X_{a}^{b c}+\sum_{b \leq c \leq d} \lambda_{b c d}^{a} X_{a}^{b c d}=0 \\
& \lambda_{a}, \lambda_{b}^{a}, \lambda_{b c}^{a}, \lambda_{b c d}^{a} \in C^{\infty}\left(J^{1}\left(M \times_{N} C\right)\right) .
\end{aligned}
$$

By applying (2.13) to $x^{a}$ (resp. $y_{a b}$ ) we obtain $\lambda_{a}=0$ (resp. $\lambda_{b}^{a}=0$ ); again by applying (2.13) to $A_{b c}^{a}, b \leq c$ (resp. $\left.A_{b c}^{a}, c \leq b\right)$ and taking the expressions of the vector fields (2.11) and (2.12) into account, we obtain $\lambda_{b c}^{a}=0, b \leq c$ (resp. $\left.\lambda_{b c}^{a}=0, c \leq b\right)$. Hence, (2.13) reads $\sum_{b \leq c \leq d} \lambda_{b c d}^{a} X_{a}^{b c d}=0$, and by applying it to $A_{b c, d}^{a}$ and taking the expressions of the vector fields (2.12) into account, we finally obtain $\lambda_{b c d}^{a}=0$. The distribution

$$
\mathcal{D}_{M \times{ }_{N} C}=\left\{\bar{X}_{\left(j_{x}^{1} g, j_{x}^{1} s_{\Gamma}\right)}^{(1)}: X \in \mathfrak{X}(N),\left(j_{x}^{1} g, j_{x}^{1} s_{\Gamma}\right) \in J^{1}\left(M \times{ }_{N} C\right)\right\}
$$

in $T\left(J^{1}\left(M \times{ }_{N} C\right)\right)$, where $\bar{X}^{(1)}$ is defined in $(2.7)$, is involutive as

$$
\left[\bar{X}^{(1)}, \bar{Y}^{(1)}\right]=\overline{[X, Y]}^{(1)}, \quad \forall X, Y \in \mathfrak{X}(N),
$$

and it is spanned by $X^{i}, X_{h}^{i}, X_{h}^{i k}, X_{i}^{j k h}$, as proved by the formulas above. The rest of the statement follows from the following identities:

$$
\begin{aligned}
\#\left\{X^{i} ; X_{h}^{i} ; X_{h}^{i k}, i \leq k ; X_{i}^{j k h}, h \leq j\right. & \leq k: h, i, j, k=1, \ldots, n\} \\
& =n+n^{2}+n\left(\begin{array}{c}
n+1 \\
2
\end{array}\right)+n\left(\begin{array}{c}
n+2 \\
3
\end{array}\right)=n\left(\begin{array}{c}
n+3 \\
3
\end{array}\right), \\
\operatorname{dim} J^{1}\left(M \times_{N} C\right)-n\left(\begin{array}{c}
n+3 \\
3
\end{array}\right)= & \frac{1}{6}\left(5 n^{4}+3 n^{3}-5 n^{2}+3 n\right) .
\end{aligned}
$$




\section{Invariance of covariant Hamiltonians}

\subsection{Position of the problem}

On the bundle $E=M \times{ }_{N} C$, an Ehresmann connection can locally be written as follows:

$$
\begin{aligned}
& \gamma=\sum_{i \leq j}\left(d y_{i j}+\gamma_{i j k} d x^{k}\right) \otimes \frac{\partial}{\partial y_{i j}}+\left(d A_{j k}^{i}+\gamma_{j k l}^{i} d x^{l}\right) \otimes \frac{\partial}{\partial A_{j k}^{i}} \\
& \gamma_{i j k}, \gamma_{j k l}^{i} \in C^{\infty}\left(M \times_{N} C\right) .
\end{aligned}
$$

In particular, for a Lagrangian density $\Lambda$ on $J^{1}\left(M \times{ }_{N} C\right)$, we obtain

$$
\begin{aligned}
\Lambda^{\gamma}= & \left(\sum_{i \leq j}\left(\gamma_{i j k}+y_{i j, k}\right) \frac{\partial L}{\partial y_{i j, k}}+\left(\gamma_{j k l}^{i}+A_{j k, l}^{i}\right) \frac{\partial L}{\partial A_{j k, l}^{i}}-L\right) \\
& \times d x^{1} \wedge \cdots \wedge d x^{n},
\end{aligned}
$$

or equivalently, $\mathcal{L}^{\gamma}=D^{\gamma}(\mathcal{L})-\mathcal{L}$, where

$$
D^{\gamma}=\sum_{i \leq j}\left(\gamma_{i j k}+y_{i j, k}\right) \frac{\partial}{\partial y_{i j, k}}+\left(\gamma_{j k l}^{i}+A_{j k, l}^{i}\right) \frac{\partial}{\partial A_{j k, l}^{i}} .
$$

Remark 3.1. The horizontal form $\left(p_{0}^{1}\right)^{*} \gamma-\theta=\left(\gamma_{i}^{\alpha}+y_{i}^{\alpha}\right) d x^{i} \otimes \partial / \partial y^{\alpha}$ can also be viewed as the $p_{0}^{1}$-vertical vector field

$$
D^{\gamma}=\left(\gamma_{i}^{\alpha}+y_{i}^{\alpha}\right) \frac{\partial}{\partial y_{i}^{\alpha}},
$$

taking the natural isomorphism $V\left(p_{0}^{1}\right) \cong\left(p_{0}^{1}\right)^{*}\left(p^{*} T^{*} N \otimes V(p)\right)$ into account (cf. $[23,24,32,34]$ ).

According to the previous formulas, this means: if the system (2.10) holds for a Lagrangian function $\mathcal{L}$, then it also holds for the covariant Hamilton$\operatorname{ian} \mathcal{L}^{\gamma}$.

If $X \in\left\{X^{i}, X_{h}^{i}, X_{h}^{i k}, X_{i}^{j k h}\right\}$, then $X\left(\mathcal{L}^{\gamma}\right)=X\left(D^{\gamma}(\mathcal{L})\right)$, as $\mathcal{L}$ is assumed to be invariant and hence $X(\mathcal{L})=0$. Therefore

$$
\begin{aligned}
X\left(\mathcal{L}^{\gamma}\right) & =X\left(D^{\gamma}(\mathcal{L})\right) \\
& =\left[X, D^{\gamma}\right](\mathcal{L}),
\end{aligned}
$$

and we conclude the following: 
Proposition 3.2. The property $(\boldsymbol{P})$ holds for an Ehresmann connection $\gamma$ on $M \times{ }_{N} C$ if and only if the vector field $D^{\gamma}$ transforms the sections of the distribution $\mathcal{D}_{M \times_{N} C}$ into themselves, namely, $\left[D^{\gamma}, \Gamma\left(\mathcal{D}_{M \times{ }_{N} C}\right)\right] \subseteq \Gamma$ $\left(\mathcal{D}_{M \times{ }_{N} C}\right)$.

The problem thus reduces to compute the brackets $\left[X^{i}, D^{\gamma}\right],\left[X_{h}^{i}, D^{\gamma}\right]$, $\left[X_{h}^{i k}, D^{\gamma}\right]$, and $\left[X_{i}^{j k h}, D^{\gamma}\right]$. We have

$$
\begin{aligned}
{\left[X^{h}, D^{\gamma}\right]=} & \sum_{i \leq j} \frac{\partial \gamma_{i j k}}{\partial x^{h}} \frac{\partial}{\partial y_{i j, k}}+\frac{\partial \gamma_{j k l}^{i}}{\partial x^{h}} \frac{\partial}{\partial A_{j k, l}^{i}} \\
{\left[X_{b}^{c d a}, D^{\gamma}\right]=} & X_{b}^{c d a}, \quad \forall b, c \leq d \leq a \\
{\left[X_{h}^{i}, D^{\gamma}\right]=} & \sum_{a \leq b} Y_{h}^{i}\left(\gamma_{a b k}\right) \frac{\partial}{\partial y_{a b, k}}+\sum_{i \leq h} \gamma_{i h k} \frac{\partial}{\partial y_{i i, k}}+\sum_{h<i} \gamma_{h i k} \frac{\partial}{\partial y_{i i, k}} \\
& +\sum_{h \leq j} \gamma_{h j k} \frac{\partial}{\partial y_{i j, k}}+\sum_{j<h} \gamma_{j h k} \frac{\partial}{\partial y_{i j, k}}+\sum_{a \leq b} \gamma_{a b h} \frac{\partial}{\partial y_{a b, i}} \\
& +\left(Y_{h}^{i}\left(\gamma_{b c r}^{a}\right)-\delta_{a}^{h} \gamma_{b c r}^{i}+\delta_{i}^{c} \gamma_{b h r}^{a}+\delta_{i}^{b} \gamma_{h c r}^{a}+\delta_{i}^{r} \gamma_{b c h}^{a}\right) \frac{\partial}{\partial A_{b c, r}^{a}} \\
{\left[X_{h}^{i k}, D^{\gamma}\right]=} & \sum_{a \leq b} Y_{h}^{i k}\left(\gamma_{a b c}\right) \frac{\partial}{\partial y_{a b, c}}+Y_{h}^{i k}\left(\gamma_{a b c}^{d}\right) \frac{\partial}{\partial A_{a b, c}^{d}}+X_{h}^{i k}-Y_{h}^{i k},
\end{aligned}
$$

where

$$
\begin{aligned}
Y_{h}^{i} & =-y_{h i} \frac{\partial}{\partial y_{i i}}-y_{h j} \frac{\partial}{\partial y_{i j}}+A_{j k}^{i} \frac{\partial}{\partial A_{j k}^{h}}-A_{j h}^{r} \frac{\partial}{\partial A_{j i}^{r}}-A_{h k}^{r} \frac{\partial}{\partial A_{i k}^{r}}, \\
Y_{h}^{i k} & =-\frac{\partial}{\partial A_{i k}^{h}}-\frac{\partial}{\partial A_{k i}^{h}}
\end{aligned}
$$

and the following formula has been used:

$$
\frac{\partial y_{r s, k}}{\partial y_{i j, h}}=\delta_{h}^{k}\left(\delta_{i}^{r} \delta_{j}^{s}+\delta_{j}^{r} \delta_{i}^{s}-\delta_{j}^{i} \delta_{r}^{i} \delta_{s}^{j}\right)
$$

\subsection{The class of the Ehresmann connections defined}

Let $p: M \times{ }_{N} C \rightarrow N, \operatorname{pr}_{1}: M \times_{N} C \rightarrow M, \operatorname{pr}_{2}: M \times_{N} C \rightarrow C$ be the natural projections. By taking the differential of $\mathrm{pr}_{1}$ and $\mathrm{pr}_{2}$, a natural 
identification is obtained $T\left(M \times_{N} C\right)=T M \times_{T N} T C$. Hence

$$
\begin{aligned}
V(p) & =V\left(p_{M}\right) \times_{N} V\left(p_{C}\right) \\
& =\operatorname{pr}_{1}^{*} V\left(p_{M}\right) \oplus \operatorname{pr}_{2}^{*} V\left(p_{C}\right)
\end{aligned}
$$

and two unique vector-bundle homomorphisms exist

$$
\gamma_{M}: \operatorname{pr}_{1}^{*} T M \rightarrow \operatorname{pr}_{1}^{*} V\left(p_{M}\right), \quad \gamma_{C}: \operatorname{pr}_{2}^{*} T C \rightarrow \operatorname{pr}_{2}^{*} V\left(p_{C}\right),
$$

such that,

$$
\begin{aligned}
\gamma(X) & =\left(\gamma_{M}\left(\operatorname{pr}_{1 *} X\right), \gamma_{C}\left(\operatorname{pr}_{2 *} X\right)\right), \quad \forall X \in T\left(M \times_{N} C\right) \\
\gamma_{M}(Y) & =Y, \quad \forall Y \in \operatorname{pr}_{1}^{*} V\left(p_{M}\right), \\
\gamma_{C}(Z) & =Z, \quad \forall Z \in \operatorname{pr}_{2}^{*} V\left(p_{C}\right) .
\end{aligned}
$$

If $\gamma$ is given by the local expression of formula (3.1), then

$$
\begin{aligned}
& \gamma_{M}=\sum_{i \leq j}\left(d y_{i j}+\gamma_{i j k} d x^{k}\right) \otimes \frac{\partial}{\partial y_{i j}}, \quad \gamma_{C}=\left(d A_{j k}^{i}+\gamma_{j k l}^{i} d x^{l}\right) \otimes \frac{\partial}{\partial A_{j k}^{i}}, \\
& \gamma_{i j k}, \gamma_{j k l}^{i} \in C^{\infty}\left(M \times_{N} C\right) .
\end{aligned}
$$

\subsubsection{The first geometric condition on $\gamma$}

Let $q: F(N) \rightarrow M$ be the projection given by

$$
\begin{aligned}
q\left(X_{1}, \ldots, X_{n}\right) & =g_{x} \\
& =\varepsilon_{h} w^{h} \otimes w^{h},
\end{aligned}
$$

where $\left(w^{1}, \ldots, w^{n}\right)$ is the dual coframe of $\left(X_{1}, \ldots, X_{n}\right) \in F_{x}(N)$, i.e., $g_{x}$ is the metric for which $\left(X_{1}, \ldots, X_{n}\right)$ is a $g_{x}$-orthonormal basis and $\varepsilon_{h}=1$ for $1 \leq h \leq n^{+}, \varepsilon_{h}=-1$ for $n^{+}+1 \leq h \leq n$. As readily seen, $q$ is a principal $G$-bundle with $G=O\left(n^{+}, n^{-}\right)$.

Given a linear connection $\Gamma$ and a tangent vector $X \in T_{x} N$, for every $u$ in $p^{-1}(x)$ there exists a unique $\Gamma$-horizontal tangent vector $X_{u}^{h_{\Gamma}} \in T_{u}(F N)$ such that, $\left(p_{F}\right)_{*} X_{u}^{h_{\Gamma}}=X$. The local expression for the horizontal lift is known to be ( [20, Chapter III, Proposition 7.4]),

$$
\left(\frac{\partial}{\partial x^{j}}\right)^{h_{\Gamma}}=\frac{\partial}{\partial x^{j}}-\Gamma_{j k}^{i} x_{l}^{k} \frac{\partial}{\partial x_{l}^{i}} .
$$


Lemma 3.3. Given a metric $g_{x} \in p_{M}^{-1}(x)$, let $u \in p_{F}^{-1}(x)$ be a linear frame such that $q(u)=g_{x}$. The projection $q_{*}\left(X_{u}^{h_{\Gamma x}}\right)$ does not depend on the linear frame $u$ chosen over $g_{x}$.

Proof. In fact, any other linear frame projecting onto $g_{x}$ can be written as $u$. $A, A \in G$. As the horizontal distribution is invariant under right translations (see [20, II, Proposition 1.2]), the following equation holds: $\left(R_{A}\right)_{*}\left(X_{u}^{h_{\Gamma}}\right)=$ $X_{u \cdot A}^{h_{\Gamma}}$. Hence

$$
\begin{aligned}
q_{*}\left(X_{u \cdot A}^{h_{\Gamma}}\right) & =q_{*}\left(\left(R_{A}\right)_{*}\left(X_{u}^{h_{\Gamma}}\right)\right) \\
& =\left(q \circ R_{A}\right)_{*}\left(X_{u}^{h_{\Gamma}}\right) \\
& =q_{*}\left(X_{u}^{h_{\Gamma}}\right) .
\end{aligned}
$$

Proposition 3.4. An Ehresmann connection $\gamma$ on $M \times{ }_{N} C$ satisfies the following condition:

$$
\left(C_{M}\right): \gamma_{M}\left(\left(g_{x}, \Gamma_{x}\right), X\right)=X-q_{*}\left(\left(\left(p_{M}\right)_{*}(X)\right)_{u}^{h_{\Gamma x}}\right),
$$

$\forall X \in T_{g_{x}} M, u \in q^{-1}\left(g_{x}\right)$, (which does not depend on the linear frame $u \in$ $q^{-1}\left(g_{x}\right)$ chosen, according to Lemma 3.3$)$ if and only if the following equations hold:

$$
\gamma_{k l j}=-\left(y_{a l} A_{j k}^{a}+y_{a k} A_{j l}^{a}\right)
$$

where the functions $\gamma_{k l j}$ (resp. $y_{i j}$, resp. $A_{j k}^{i}$ ) are defined in the formula (3.1) (resp. (2.2), resp. (2.4)).

Proof. Letting $\left(\chi_{j}^{i}\right)_{i, j=1}^{n}=\left(\left(x_{j}^{i}\right)_{i, j=1}^{n}\right)^{-1}$, the dual coframe of the linear frame $u=\left(X_{1}, \ldots, X_{n}\right) \in F_{x}(N)$ given in $(2.3)$ is $\left(w^{1}, \ldots, w^{n}\right), w^{h}=\chi_{k}^{h}(u)$ $\left(d x^{k}\right)_{x}, 1 \leq h \leq n$, and the projection $q$ is given by

$$
\begin{aligned}
q(u) & =g_{x} \\
& =\sum_{h=1}^{n} \varepsilon_{h} \chi_{k}^{h}(u) \chi_{l}^{h}(u)\left(d x^{k}\right)_{x} \otimes\left(d x^{l}\right)_{x} .
\end{aligned}
$$


Therefore the equations of the projection (3.6) are as follows:

$$
\begin{aligned}
x^{i} \circ q & =x^{i}, \\
y_{k l} \circ q & =\sum_{h=1}^{n} \varepsilon_{h} \chi_{k}^{h} \chi_{l}^{h} .
\end{aligned}
$$

Hence

$$
q_{*}\left(\frac{\partial}{\partial x_{b}^{a}}\right)_{u}=\sum_{k \leq l} \varepsilon_{h}\left\{\frac{\partial \chi_{k}^{h}}{\partial x_{b}^{a}} \chi_{l}^{h}+\chi_{k}^{h} \frac{\partial \chi_{l}^{h}}{\partial x_{b}^{a}}\right\}(u)\left(\frac{\partial}{\partial y_{k l}}\right)_{g_{x}} .
$$

Taking derivatives with respect to $x_{b}^{a}$ on the identity $\chi_{r}^{h} x_{i}^{r}=\delta_{i}^{h}$, multiplying the outcome by $\chi_{k}^{i}$, and summing up over the index $i$, the following formula is obtained: $\partial \chi_{k}^{h} / \partial x_{b}^{a}=-\chi_{a}^{h} \chi_{k}^{b}$. Replacing this equation into the expression for $q_{*}\left(\partial / \partial x_{b}^{a}\right)_{u}$ above, we have

$$
q_{*}\left(\frac{\partial}{\partial x_{b}^{a}}\right)_{u}=-\sum_{k \leq l}\left\{\chi_{k}^{b}(u) y_{a l}\left(g_{x}\right)+\chi_{l}^{b}(u) y_{a k}\left(g_{x}\right)\right\}\left(\frac{\partial}{\partial y_{k l}}\right)_{g_{x}} .
$$

From (3.7), evaluated at $u \in q^{-1}\left(g_{x}\right)$, we deduce

$$
\begin{aligned}
q_{*}\left(\frac{\partial}{\partial x^{j}}\right)_{u}^{h_{\Gamma}}= & \left(\frac{\partial}{\partial x^{j}}\right)_{g_{x}}-\Gamma_{j c}^{a}(x) x_{b}^{c}(u) q_{*}\left(\frac{\partial}{\partial x_{b}^{a}}\right)_{g_{x}} \\
= & \left(\frac{\partial}{\partial x^{j}}\right)_{g_{x}} \\
& +\sum_{k \leq l} \Gamma_{j c}^{a}(x) x_{b}^{c}(u)\left\{\chi_{k}^{b}(u) y_{a l}\left(g_{x}\right)+\chi_{l}^{b}(u) y_{a k}\left(g_{x}\right)\right\} \\
& \times\left(\frac{\partial}{\partial y_{k l}}\right)_{g_{x}} \\
= & \left(\frac{\partial}{\partial x^{j}}\right)_{g_{x}}+\sum_{k \leq l}\left\{\Gamma_{j k}^{a}(x) y_{a l}\left(g_{x}\right)+\Gamma_{j l}^{a}(x) y_{a k}\left(g_{x}\right)\right\}\left(\frac{\partial}{\partial y_{k l}}\right)_{g_{x}} .
\end{aligned}
$$

The condition $\left(C_{M}\right)$ holds automatically whenever $X \in V\left(p_{M}\right)$. Hence, $\left(C_{M}\right)$ holds if and only if it holds for $X=\left(\partial / \partial x^{j}\right)_{g_{x}}$, namely,

$$
\begin{aligned}
\sum_{k \leq l} \gamma_{k l j}\left(g_{x}, \Gamma_{x}\right)\left(\frac{\partial}{\partial y_{k l}}\right)_{g_{x}} & =\gamma_{M}\left(\left(g_{x}, \Gamma_{x}\right),\left(\frac{\partial}{\partial x^{j}}\right)_{g_{x}}\right) \\
& =\left(\frac{\partial}{\partial x^{j}}\right)_{g_{x}}-q_{*}\left(\frac{\partial}{\partial x^{j}}\right)_{u}^{h_{\Gamma x}}
\end{aligned}
$$




$$
\begin{aligned}
= & -\sum_{k \leq l}\left\{\Gamma_{j k}^{a}(x) y_{a l}\left(g_{x}\right)+\Gamma_{j l}^{a}(x) y_{a k}\left(g_{x}\right)\right\} \\
& \times\left(\frac{\partial}{\partial y_{k l}}\right)_{g_{x}},
\end{aligned}
$$

thus proving formula (3.8) in the statement.

\subsubsection{The canonical covariant derivative}

As is known (e.g., see [20, III, Section 1], [23, pp. 157-158]) every connection $\Gamma$ on a principal $G$-bundle $P \rightarrow N$ induces a covariant derivative $\nabla^{\Gamma}$ on the vector bundle associated to $P$ under a linear representation $\rho: G \rightarrow G l(m, \mathbb{R})$ with standard fibre $\mathbb{R}^{m}$. In particular, this applies to the principal bundle of linear frames, thus proving that every linear connection $\Gamma$ on $N$ induces a covariant derivative $\nabla^{\Gamma}$ on every tensorial vector bundle $E \rightarrow N$.

The bundles $\left(p_{C}\right)^{*} E$, where $E$ is a tensorial vector bundle, are endowed with a canonical covariant derivative $\nabla^{E}$ completely determined by the formula:

$$
\left(\left(\nabla^{E}\right)_{X}(f \xi)\right)\left(\Gamma_{x}\right)=((X f) \xi)\left(\Gamma_{x}\right)+f\left(\Gamma_{x}\right)\left(\nabla_{\left(p_{C}\right)_{*} X}^{\Gamma_{x}} \xi\right)(x),
$$

for all $X \in T_{\Gamma_{x}} C, f \in C^{\infty}(C)$, and every local section $\xi$ of $E$ defined on a neighbourhood of $x$. The uniqueness of $\nabla^{E}$ follows from (3.9) as the sections of $E$ span the sections of $\left(p_{C}\right)^{*} E$ over $C^{\infty}(C)$, see [8, 0.3.6]. Below, we are specially concerned with the cases $E=T N$ and $E=\wedge^{2} T^{*} N \otimes T N$.

\subsubsection{The 2-form associated with $\gamma_{C}$}

As $p_{C}: C \rightarrow N$ is an affine bundle modelled over $\otimes^{2} T^{*} N \otimes T N$, there is a natural identification

$$
V\left(p_{C}\right) \cong\left(p_{C}\right)^{*}\left(\otimes^{2} T^{*} N \otimes T N\right)
$$

and consequently, an Ehresmann connection $\gamma_{C}$ on $C$ can also be viewed as a homomorphism $\gamma_{C}: T C \rightarrow \otimes^{2} T^{*} N \otimes T N$. If $\gamma_{C}$ is locally given by

$$
\gamma_{C}=\left(d A_{j k}^{i}+\gamma_{j k l}^{i} d x^{l}\right) \otimes \frac{\partial}{\partial A_{j k}^{i}}, \quad \gamma_{j k l}^{i} \in C^{\infty}(C),
$$

then

$$
\gamma_{C}=\left(d A_{j k}^{i}+\gamma_{j k l}^{i} d x^{l}\right) \otimes d x^{j} \otimes d x^{k} \otimes \frac{\partial}{\partial x^{i}},
$$


and $\gamma_{C}$ induces a 2-form $\tilde{\gamma}_{C}$ taking values in $\left(p_{C}\right)^{*}\left(T^{*} N \otimes T N\right)$ as follows:

$$
\begin{aligned}
& \tilde{\gamma}_{C}(X, Y)=c_{1}^{1}\left(\left(p_{C}\right)_{*}(Y) \otimes \gamma_{C}(X)\right)-c_{1}^{1}\left(\left(p_{C}\right)_{*}(X) \otimes \gamma_{C}(Y)\right), \\
& \forall X, Y \in T_{\Gamma_{x}} C,
\end{aligned}
$$

where

$$
\begin{aligned}
& c_{1}^{1}: T N \otimes T^{*} N \otimes T^{*} N \otimes T N \rightarrow T^{*} N \otimes T N, \\
& c_{1}^{1}\left(X_{1} \otimes w_{1} \otimes w_{2} \otimes X_{2}\right)=w_{1}\left(X_{1}\right) w_{2} \otimes X_{2}, \\
& X_{1}, X_{2} \in T_{x} N, w_{1}, w_{2} \in T_{x}^{*} N .
\end{aligned}
$$

If $\gamma_{C}$ is given by (3.10), then from the very definition of $\tilde{\gamma}_{C}$ the following local expression is obtained:

$$
\tilde{\gamma}_{C}=\left(d A_{l h}^{c}+\left(\gamma_{l h a}^{c}-\gamma_{a h l}^{c}\right) d x^{a}\right) \wedge d x^{l} \otimes d x^{h} \otimes \frac{\partial}{\partial x^{c}}
$$

\subsubsection{The second geometric condition on $\gamma$}

Let alt $_{12}: \otimes^{2} T^{*} N \otimes T N \rightarrow \wedge^{2} T^{*} N \otimes T N$ be the operator alternating the two covariant arguments.

The vector bundle $\left(p_{C}\right)^{*}\left(\wedge^{2} T^{*} N \otimes T N\right)$ admits a canonical section

$$
\begin{gathered}
\tau_{N}: C \rightarrow \wedge^{2} T^{*} N \otimes T N, \\
\tau_{N}\left(\Gamma_{x}\right)=T^{\Gamma x}, \quad \forall \Gamma_{x} \in C,
\end{gathered}
$$

where $T^{\Gamma x}$ is the torsion of $\Gamma_{x}$. Locally,

$$
\tau_{N}=\sum_{j<k}\left(A_{j k}^{i}-A_{k j}^{i}\right) d x^{j} \wedge d x^{k} \otimes \frac{\partial}{\partial x^{i}}
$$

From the previous formulas the next result follows:

Proposition 3.5. Let $\gamma$ be an Ehresmann connection on $M \times{ }_{N} C$, let $\nabla^{(1)}=\nabla^{E_{1}}$ with $E_{1}=T N$, let $R^{\nabla^{(1)}}$ be its curvature form, and finally, let $\nabla^{(2)}=\nabla^{E_{2}}$ with $E_{2}=\wedge^{2} T^{*} N \otimes T N$. 
$\left(C_{C}\right)$ Assume the component $\gamma_{C}$ of $\gamma$ is defined on $C$. Then, the equations

$$
\begin{aligned}
\tilde{\gamma}_{C} & =R^{\nabla^{(1)}}, \\
\operatorname{alt}_{12} \circ \gamma_{C} & =\nabla^{(2)} \tau_{N},
\end{aligned}
$$

are locally equivalent to the following ones:

$$
\begin{aligned}
\gamma_{s t r}^{h}-\gamma_{r t s}^{h}= & A_{r m}^{h} A_{s t}^{m}-A_{s m}^{h} A_{r t}^{m}, \\
\gamma_{r s t}^{h}-\gamma_{s r t}^{h}= & A_{t m}^{h}\left(A_{r s}^{m}-A_{s r}^{m}\right)+A_{t s}^{m}\left(A_{m r}^{h}-A_{r m}^{h}\right) \\
& +A_{t r}^{m}\left(A_{s m}^{h}-A_{m s}^{h}\right) .
\end{aligned}
$$

\subsection{Solution to the problem (P)}

Theorem 3.6. If the connection $\gamma$ on $M \times{ }_{N} C$ satisfies the conditions $\left(C_{M}\right)$ and $\left(C_{C}\right)$ introduced above, then the vector field $D^{\gamma}$ satisfies the property stated in Proposition 3.2 and, accordingly the covariant Hamiltonian with respect to $\gamma$ of every $\mathfrak{X}(N)$-invariant Lagrangian is also $\mathfrak{X}(N)$-invariant.

Proof. When $\gamma_{M}$ satisfies the condition $\left(C_{M}\right)$ the brackets (3.3), (3.4), and (3.5) are respectively given by

$$
\begin{aligned}
{\left[X^{h}, D^{\gamma}\right]=} & \frac{\partial \gamma_{j k l}^{i}}{\partial x^{h}} \frac{\partial}{\partial A_{j k, l}^{i}}, \\
{\left[X_{h}^{i}, D^{\gamma}\right]=} & \left(Y_{h}^{i}\left(\gamma_{b c r}^{a}\right)-\delta_{a}^{h} \gamma_{b c r}^{i}+\delta_{i}^{c} \gamma_{b h r}^{a}+\delta_{i}^{b} \gamma_{h c r}^{a}+\delta_{i}^{r} \gamma_{b c h}^{a}\right) \frac{\partial}{\partial A_{b c, r}^{a}} \\
{\left[X_{h}^{i k}, D^{\gamma}\right]=} & \left(-\frac{\partial \gamma_{a b c}^{d}}{\partial A_{i k}^{h}}+\delta_{i}^{c}\left(\delta_{d}^{h} A_{a b}^{k}-\delta_{b}^{k} A_{a h}^{d}-\delta_{a}^{k} A_{h b}^{d}\right)\right. \\
& \left.-\frac{\partial \gamma_{a b c}^{d}}{\partial A_{k i}^{h}}+\delta_{k}^{c}\left(\delta_{d}^{h} A_{a b}^{i}-\delta_{b}^{i} A_{a h}^{d}-\delta_{a}^{i} A_{h b}^{d}\right)\right) \frac{\partial}{\partial A_{a b, c}^{d}}
\end{aligned}
$$

In addition, if $\gamma_{C}$ satisfies the condition $\left(C_{C}\right)$, then taking derivatives with respect to $x^{h}$ in (3.13) and (3.14), we obtain

$$
\frac{\partial \gamma_{k l j}^{i}}{\partial x^{h}}=\frac{\partial \gamma_{j l k}^{i}}{\partial x^{h}}, \quad \frac{\partial \gamma_{j k l}^{i}}{\partial x^{h}}=\frac{\partial \gamma_{k j l}^{i}}{\partial x^{h}}
$$


and renaming indices we deduce

$$
\begin{aligned}
& \frac{\partial \gamma_{j j k}^{i}}{\partial x^{h}}=\frac{\partial \gamma_{j k j}^{i}}{\partial x^{h}}=\frac{\partial \gamma_{k j j}^{i}}{\partial x^{h}}(j<k), \\
& \frac{\partial \gamma_{k k j}^{i}}{\partial x^{h}}=\frac{\partial \gamma_{k j k}^{i}}{\partial x^{h}}=\frac{\partial \gamma_{j k k}^{i}}{\partial x^{h}}(j<k), \\
& \frac{\partial \gamma_{j k l}^{i}}{\partial x^{h}}=\frac{\partial \gamma_{k l j}^{i}}{\partial x^{h}}=\frac{\partial \gamma_{l j k}^{i}}{\partial x^{h}}=\frac{\partial \gamma_{k j l}^{i}}{\partial x^{h}}=\frac{\partial \gamma_{l k j}^{i}}{\partial x^{h}}=\frac{\partial \gamma_{j l k}^{i}}{\partial x^{h}}(j<k<l) .
\end{aligned}
$$

From (3.15) we obtain

$$
\begin{aligned}
{\left[X^{h}, D^{\gamma}\right]=} & \sum_{j<k<l} \frac{\partial \gamma_{j k l}^{i}}{\partial x^{h}} X_{i}^{j k l}+\frac{1}{2} \sum_{j<k} \frac{\partial \gamma_{j j k}^{i}}{\partial x^{h}} X_{i}^{j j k} \\
& +\frac{1}{2} \sum_{j<k} \frac{\partial \gamma_{k k j}^{i}}{\partial x^{h}} X_{i}^{k k j}+\frac{1}{6} \frac{\partial \gamma_{j j j}^{i}}{\partial x^{h}} X_{i}^{j j j}
\end{aligned}
$$

and consequently the values of $\left[X^{h}, D^{\gamma}\right]$ belong to the distribution $\mathcal{D}_{M \times{ }_{N} C}$.

Moreover, as $\gamma_{C}$ is assumed to be defined on $C$, we have

$$
Y_{h}^{i}\left(\gamma_{b c r}^{a}\right)=\left(\delta_{h}^{s} A_{j k}^{i}-\delta_{k}^{i} A_{j h}^{s}-\delta_{j}^{i} A_{h k}^{s}\right) \frac{\partial \gamma_{b c r}^{a}}{\partial A_{j k}^{s}}
$$

For the sake of simplicity, below we set

$$
\left(T_{h}^{i}\right)_{b c r}^{a}=A_{j k}^{i} \frac{\partial \gamma_{b c r}^{a}}{\partial A_{j k}^{h}}-A_{j h}^{s} \frac{\partial \gamma_{b c r}^{a}}{\partial A_{j i}^{s}}-A_{h k}^{s} \frac{\partial \gamma_{b c r}^{a}}{\partial A_{i k}^{s}}-\delta_{a}^{h} \gamma_{b c r}^{i}+\delta_{i}^{b} \gamma_{h c r}^{a}+\delta_{i}^{c} \gamma_{b h r}^{a}+\delta_{i}^{r} \gamma_{b c h}^{a}
$$

Taking derivatives with respect to $A_{j k}^{s}$, equations (3.13) and (3.14) yield

$$
\begin{aligned}
\frac{\partial \gamma_{b c r}^{a}}{\partial A_{j k}^{s}}-\frac{\partial \gamma_{r c b}^{a}}{\partial A_{j k}^{s}}= & \delta_{r}^{j} \delta_{s}^{a} A_{b c}^{k}-\delta_{b}^{j} \delta_{s}^{a} A_{r c}^{k}+\delta_{b}^{j} \delta_{C}^{k} A_{r s}^{a}-\delta_{r}^{j} \delta_{C}^{k} A_{b s}^{a}, \\
\frac{\partial \gamma_{r b c}^{a}}{\partial A_{j k}^{s}}-\frac{\partial \gamma_{b r c}^{a}}{\partial A_{j k}^{s}}= & \delta_{c}^{j} \delta_{s}^{a} A_{r b}^{k}-\delta_{s}^{a} \delta_{c}^{j} A_{b r}^{k}-\delta_{s}^{a} \delta_{b}^{k} A_{c r}^{j}-\delta_{s}^{a} \delta_{r}^{j} A_{c b}^{k}+\delta_{s}^{a} \delta_{r}^{k} A_{c b}^{j}+\delta_{s}^{a} \delta_{b}^{j} A_{c r}^{k} \\
& +\delta_{c}^{j} \delta_{b}^{k} A_{s r}^{a}-\delta_{c}^{j} \delta_{r}^{k} A_{s b}^{a}+\delta_{r}^{j} \delta_{b}^{k} A_{c s}^{a}-\delta_{b}^{j} \delta_{r}^{k} A_{c s}^{a}+\delta_{c}^{j} \delta_{r}^{k} A_{b s}^{a} \\
& -\delta_{c}^{j} \delta_{b}^{k} A_{r s}^{a} .
\end{aligned}
$$


From these expressions, the following symmetries of indices are obtained:

$$
\begin{aligned}
& \left(T_{h}^{i}\right)_{b b c}^{a}=\left(T_{h}^{i}\right)_{b c b}^{a}=\left(T_{h}^{i}\right)_{c b b}^{a}(b<c), \\
& \left(T_{h}^{i}\right)_{b c c}^{a}=\left(T_{h}^{i}\right)_{c b c}^{a}=\left(T_{h}^{i}\right)_{c c b}^{a}(b<c), \\
& \left(T_{h}^{i}\right)_{b c d}^{a}=\left(T_{h}^{i}\right)_{d b c}^{a}=\left(T_{h}^{i}\right)_{c d b}^{a}=\left(T_{h}^{i}\right)_{b d c}^{a}=\left(T_{h}^{i}\right)_{d c b}^{a}=\left(T_{h}^{i}\right)_{c b d}^{a}(b<c<d),
\end{aligned}
$$

and from (3.16) we obtain

$$
\begin{aligned}
{\left[X_{h}^{i}, D^{\gamma}\right]=} & \sum_{b<c<d}\left(T_{h}^{i}\right)_{b c d}^{a} X_{a}^{b c d}+\frac{1}{2} \sum_{b<c}\left(T_{h}^{i}\right)_{b b c}^{a} X_{a}^{b b c} \\
& +\frac{1}{2} \sum_{b<c}\left(T_{h}^{i}\right)_{c c b}^{a} X_{a}^{c c b}+\frac{1}{6}\left(T_{h}^{i}\right)_{b b b}^{a} X_{a}^{b b b} .
\end{aligned}
$$

Hence $\left[X_{h}^{i}, D^{\gamma}\right]$ also takes values into the distribution $\mathcal{D}_{M \times{ }_{N} C}$.

The proof for the third bracket is similar to the previous two cases but longer. Letting

$$
\begin{aligned}
\left(T_{h}^{i k}\right)_{r b c}^{a}= & -\frac{\partial \gamma_{r b c}^{a}}{\partial A_{i k}^{h}}-\frac{\partial \gamma_{r b c}^{a}}{\partial A_{k i}^{h}}+\delta_{i}^{c}\left(\delta_{a}^{h} A_{r b}^{k}-\delta_{b}^{k} A_{r h}^{a}-\delta_{r}^{k} A_{h b}^{a}\right) \\
& +\delta_{k}^{c}\left(\delta_{a}^{h} A_{r b}^{i}-\delta_{b}^{i} A_{r h}^{a}-\delta_{r}^{i} A_{h b}^{a}\right),
\end{aligned}
$$

the following symmetries are obtained:

$$
\begin{aligned}
\left(T_{h}^{i k}\right)_{b b c}^{a}= & \left(T_{h}^{i k}\right)_{b c b}^{a}=\left(T_{h}^{i k}\right)_{c b b}^{a}(b<c), \\
\left(T_{h}^{i k}\right)_{b c c}^{a}= & \left(T_{h}^{i k}\right)_{c b c}^{a}=\left(T_{h}^{i k}\right)_{c c b}^{a}(b<c), \\
\left(T_{h}^{i k}\right)_{b c d}^{a}= & \left(T_{h}^{i k}\right)_{d b c}^{a}=\left(T_{h}^{i k}\right)_{c d b}^{a}=\left(T_{h}^{i k}\right)_{b d c}^{a}=\left(T_{h}^{i k}\right)_{d c b}^{a}=\left(T_{h}^{i k}\right)_{c b d}^{a} \\
& (b<c<d) .
\end{aligned}
$$

Hence

$$
\begin{aligned}
{\left[X_{h}^{i k}, D^{\gamma}\right]=} & \sum_{b<c<d}\left(T_{h}^{i k}\right)_{b c d}^{a} X_{a}^{b c d}+\frac{1}{2} \sum_{b<c}\left(T_{h}^{i k}\right)_{b b c}^{a} X_{a}^{b b c} \\
& +\frac{1}{2} \sum_{b<c}\left(T_{h}^{i k}\right)_{c c b}^{a} X_{a}^{c c b}+\frac{1}{6}\left(T_{h}^{i k}\right)_{b b b}^{a} X_{a}^{b b b}
\end{aligned}
$$

and the proof is complete. 
Theorem 3.7. The Ehresmann connections on $C$ satisfying equations (3.11) and (3.12) are the sections of an affine bundle over $C$ modelled over the vector bundle $\left(p_{C}\right)^{*}\left(S^{3} T^{*} N \otimes T N\right)$. Consequently, there always exist Ehresmann connections on $M \times{ }_{N} C$ fulfilling the conditions $\left(C_{M}\right)$ and $\left(C_{C}\right)$ introduced above.

Proof. If two Ehresmann connections $\gamma_{C}, \gamma_{C}^{\prime}$ satisfy equations (3.11) and (3.12), then the difference tensor field $t=\gamma_{C}^{\prime}-\gamma_{C}$, which is a section of the bundle $\left(p_{C}\right)^{*}\left(\otimes^{3} T^{*} N \otimes T N\right)$, satisfies the following symmetries:

$$
\begin{aligned}
& t\left(X_{1}, X_{2}, X_{3}\right)=t\left(X_{3}, X_{2}, X_{1}\right), \\
& t\left(X_{1}, X_{2}, X_{3}\right)=t\left(X_{2}, X_{1}, X_{3}\right),
\end{aligned}
$$

according to (3.13) and (3.14), respectively, for all $X_{1}, X_{2}, X_{3} \in T_{x} N, \Gamma_{x} \in$ $C_{x}(N)$. Hence

$$
t\left(X_{1}, X_{3}, X_{2}\right) \stackrel{(3.17)}{=} t\left(X_{2}, X_{3}, X_{1}\right) \stackrel{(3.18)}{=} t\left(X_{3}, X_{2}, X_{1}\right) \stackrel{(3.17)}{=} t\left(X_{1}, X_{2}, X_{3}\right),
$$

thus proving that $t$ is totally symmetric. The second part of the statement thus follows from the fact that an affine bundle always admits global sections, e.g., see [20, I, Theorem 5.7].

Remark 3.8. The results obtained above also hold if the bundle of linear connections is replaced by the subbundle $C^{\mathrm{sym}}=C^{\mathrm{sym}}(N) \subset C$ of symmetric linear connections; the only difference to be observed between both bundles is that in the symmetric cases equation (3.12), or equivalently (3.14), holds automatically.

\section{The second-order formalism}

In this section we consider the problem of invariance of covariant Hamiltonians for second-order Lagrangians defined on the bundle of metrics, i.e., for functions $\mathcal{L} \in C^{\infty}\left(J^{2} M\right)$, where $M$ denotes, as throughout this paper, the bundle of pseudo-Riemannian metrics of a given signature $\left(n^{+}, n^{-}\right)$on $N$.

\subsection{Second-order Ehresmann connections}

A second-order Ehresmann connection on $p: E \rightarrow N$ is a differential 1form $\gamma^{2}$ on $J^{1} E$ taking values in the vertical sub-bundle $V\left(p^{1}\right)$ such that $\gamma^{2}(X)=X$ for every $X \in V\left(p^{1}\right)$. (We refer the reader to [29] for the basics 
on Ehresmann connections of arbitrary order.) Once a connection $\gamma^{2}$ is given, we have a decomposition of vector bundles $T\left(J^{1} E\right)=V\left(p^{1}\right) \oplus \operatorname{ker} \gamma^{2}$, where $\operatorname{ker} \gamma^{2}$ is called the horizontal sub-bundle determined by $\gamma^{2}$. In the coordinate system on $J^{1} E$ induced from a fibred coordinate system $\left(x^{j}, y^{\alpha}\right)$ for $p$, a connection form can be written as

$$
\gamma^{2}=\left(d y^{\alpha}+\gamma_{j}^{\alpha} d x^{j}\right) \otimes \frac{\partial}{\partial y^{\alpha}}+\left(d y_{i}^{\alpha}+\gamma_{i j}^{\alpha} d x^{j}\right) \otimes \frac{\partial}{\partial y_{i}^{\alpha}}, \quad \gamma_{j}^{\alpha}, \gamma_{i j}^{\alpha} \in C^{\infty}\left(J^{1} E\right)
$$

As in the first-order case, the action of the group $\operatorname{Aut}(p)$ on the space of second-order connections is defined by the formula

$$
\Phi \cdot \gamma^{2}=\left(\Phi^{(1)}\right)_{*} \circ \gamma^{2} \circ\left(\Phi^{(1)}\right)_{*}^{-1}, \quad \forall \Phi \in \operatorname{Aut}(p) .
$$

As $\Phi^{(1)}: J^{1} M \rightarrow J^{1} M$ is a morphism of fibred manifolds over $N,\left(\Phi^{(1)}\right)_{*}$ transforms the vertical subbundle $V\left(p^{1}\right)$ into itself; hence the previous definition makes sense.

\subsection{A remarkable isomorphism}

Theorem 4.1. Let $\Gamma^{g}$ be the Levi-Civita connection of a pseudoRiemannian metric $g$ on $N$. The mapping $\zeta_{N}: J^{1} M \rightarrow M \times{ }_{N} C^{\mathrm{sym}}$, $\zeta_{N}\left(j_{x}^{1} g\right)=\left(g_{x}, \Gamma_{x}^{g}\right)$ is a diffeomorphism. There is a natural one-to-one correspondence between first-order Ehresmann connections on the bundle $p$ : $M \times{ }_{N} C^{\mathrm{sym}} \rightarrow N$ and second-order Ehresmann connections on the bundle $p_{M}: M \rightarrow N$, which is explicitly given by,

$$
\gamma^{2}=\left(\left(\zeta_{N}^{v}\right)_{*}\right)^{-1} \circ \gamma \circ\left(\zeta_{N}\right)_{*}
$$

where $\gamma: T\left(M \times{ }_{N} C^{\mathrm{sym}}\right) \rightarrow V(p)$ is a first-order Ehresmann connection,

$$
\left(\zeta_{N}\right)_{*}: T\left(J^{1} M\right) \rightarrow T\left(M \times{ }_{N} C^{\mathrm{sym}}\right)
$$

is the Jacobian mapping induced by $\zeta_{N}$, and $\left(\zeta_{N}^{v}\right)_{*}: V\left(p_{M}^{1}\right) \rightarrow V(p)$ is its restriction to the vertical bundles. 
Proof. As a computation shows, the equations of $\zeta_{N}$ in the coordinate systems introduced in Section 2.1.2, are as follows:

$$
\begin{aligned}
x^{i} \circ \zeta_{N} & =x^{i}, \\
y_{i j} \circ \zeta_{N} & =y_{i j}, \\
A_{i j}^{h} \circ \zeta_{N} & =\frac{1}{2} y^{h k}\left(y_{i k, j}+y_{j k, i}-y_{i j, k}\right), \quad i \leq j,
\end{aligned}
$$

where $\left(y^{i j}\right)_{i, j=1}^{n}$ is the inverse mapping of the matrix $\left(y_{i j}\right)_{i, j=1}^{n}$ and the functions $y_{i j}$ are defined in (2.2). Hence

$$
\begin{aligned}
x^{i} \circ \zeta_{N}^{-1} & =x^{i}, \\
y_{i j} \circ \zeta_{N}^{-1} & =y_{i j}, \\
y_{i j, k} \circ \zeta_{N}^{-1} & =y_{h i} A_{j k}^{h}+y_{h j} A_{i k}^{h}, \quad i \leq j .
\end{aligned}
$$

As the diffeomorphism $\zeta_{N}$ induces the identity on the ground manifold $N$, it follows that the definition of $\gamma^{2}$ in (4.2) makes sense and the following formulas are obtained:

$$
\begin{aligned}
\gamma^{2}\left(\frac{\partial}{\partial x^{r}}\right)= & \sum_{a \leq b}\left(\gamma_{a b r} \circ \zeta_{N}\right) \frac{\partial}{\partial y_{a b}}+\sum_{i \leq j} \gamma_{i j k r} \frac{\partial}{\partial y_{i j, k}}, \\
\gamma_{i j k r}= & \frac{1}{2} \sum_{a \leq b} \frac{\delta_{a h} \delta_{b i}+\delta_{a i} \delta_{b h}}{1+\delta_{h i}}\left(\gamma_{a b r} \circ \zeta_{N}\right) y^{h l}\left(y_{j l, k}+y_{k l, j}-y_{j k, l}\right) \\
& +\frac{1}{2} \sum_{a \leq b} \frac{\delta_{a h} \delta_{b j}+\delta_{a j} \delta_{b h}}{1+\delta_{h j}}\left(\gamma_{a b r} \circ \zeta_{N}\right) y^{h l}\left(y_{i l, k}+y_{k l, i}-y_{i k, l}\right) \\
& +\sum_{j \leq a} \frac{\delta_{a k}}{1+\delta_{j k}}\left(\gamma_{j a r}^{h} \circ \zeta_{N}\right) y_{h i}+\sum_{a \leq j} \frac{\delta_{a k}}{1+\delta_{j k}}\left(\gamma_{a j r}^{h} \circ \zeta_{N}\right) y_{h i} \\
& +\sum_{i \leq a} \frac{\delta_{a k}}{1+\delta_{i k}}\left(\gamma_{i a r}^{h} \circ \zeta_{N}\right) y_{h j}+\sum_{a \leq i} \frac{\delta_{a k}}{1+\delta_{i k}}\left(\gamma_{a i r}^{h} \circ \zeta_{N}\right) y_{h j},
\end{aligned}
$$

where

$$
\gamma=\sum_{i \leq j}\left(d y_{i j}+\gamma_{i j k} d x^{k}\right) \otimes \frac{\partial}{\partial y_{i j}}+\sum_{j \leq k}\left(d A_{j k}^{i}+\gamma_{j k l}^{i} d x^{l}\right) \otimes \frac{\partial}{\partial A_{j k}^{i}},
$$

or equivalently,

$$
\gamma=\frac{1}{2-\delta_{i j}}\left(d y_{i j}+\gamma_{i j k} d x^{k}\right) \otimes \frac{\partial}{\partial y_{i j}}+\frac{1}{2-\delta_{j k}}\left(d A_{j k}^{i}+\gamma_{j k l}^{i} d x^{l}\right) \otimes \frac{\partial}{\partial A_{j k}^{i}},
$$


assuming $\gamma_{h i r}=\gamma_{i h r}$ for $h>i$, and $\gamma_{j k r}^{h}=\gamma_{k j r}^{h}$ for $j>k$. Taking the symmetry $A_{j k}^{i}=A_{k j}^{i}$ into account, we obtain

$$
\begin{aligned}
\gamma_{i j k r}= & \frac{1}{2}\left(\gamma_{h i r} \circ \zeta_{N}\right) y^{h l}\left(y_{j l, k}+y_{k l, j}-y_{j k, l}\right) \\
& +\frac{1}{2}\left(\gamma_{h j r} \circ \zeta_{N}\right) y^{h l}\left(y_{i l, k}+y_{k l, i}-y_{i k, l}\right) \\
& +\left(\gamma_{j k r}^{h} \circ \zeta_{N}\right) y_{h i}+\left(\gamma_{i k r}^{h} \circ \zeta_{N}\right) y_{h j} .
\end{aligned}
$$

Hence

$$
\gamma_{i j k r} \circ \zeta_{N}^{-1}=\gamma_{h i r} A_{j k}^{h}+\gamma_{h j r} A_{i k}^{h}+\gamma_{j k r}^{h} y_{h i}+\gamma_{i k r}^{h} y_{h j}, i \leq j .
$$

Permuting the indices $i, j, k$ cyclically on the previous equation, we have

$$
\gamma_{i j r}^{s}=-\gamma_{h k r} A_{i j}^{h} y^{k s}-\frac{1}{2}\left(\gamma_{i j k r} \circ \zeta_{N}^{-1}-\gamma_{j k i r} \circ \zeta_{N}^{-1}-\gamma_{k i j r} \circ \zeta_{N}^{-1}\right) y^{k s},
$$

thus proving that the mapping $\gamma \mapsto \gamma^{2}$ defined in the statement, is bijective.

\subsection{Covariant Hamiltonians for second-order Lagrangians}

The Legendre form of a second-order Lagrangian density $\Lambda=L v_{n}$ on the bundle $p: E \rightarrow N$ is the $V^{*}\left(p^{1}\right)$-valued $p^{3}$-horizontal $(n-1)$-form $\omega_{\Lambda}$ on $J^{3} E$ locally given by (e.g., see $\left.[17,26,35]\right)$,

$$
\omega_{\Lambda}=i_{\partial / \partial x^{i}} v_{n} \otimes\left(L_{\alpha}^{i 0} d y^{\alpha}+L_{\alpha}^{i j} d y_{j}^{\alpha}\right),
$$

where

$$
\begin{aligned}
L_{\alpha}^{i j} & =\frac{1}{2-\delta_{i j}} \frac{\partial L}{\partial y_{i j}^{\alpha}}, \\
L_{\alpha}^{i} & =\frac{\partial L}{\partial y_{i}^{\alpha}}-\sum_{j} \frac{1}{2-\delta_{i j}} D_{j}\left(\frac{\partial L}{\partial y_{i j}^{\alpha}}\right),
\end{aligned}
$$

and

$$
D_{j}=\frac{\partial}{\partial x^{j}}+\sum_{I \in \mathbb{N}^{n},|I|=0}^{\infty} y_{I+(j)}^{\alpha} \frac{\partial}{\partial y_{I}^{\alpha}}
$$

denotes the total derivative with respect to the variable $x^{j}$.

The Poincaré-Cartan form attached to $\Lambda$ is then defined to be the ordinary $n$-form on $J^{3} E$ given by, $\Theta_{\Lambda}=\left(p_{2}^{3}\right)^{*} \theta^{2} \wedge \omega_{\Lambda}+\Lambda$, where $\theta^{2}$ is the 
second-order structure form (cf. $[33,(0.36)]$ ) and the exterior product of $\left(p_{2}^{3}\right)^{*} \theta^{2}$ and the Legendre form, is taken with respect to the pairing induced by duality, $V\left(p^{1}\right) \times{ }_{J^{1} E} V^{*}\left(p^{1}\right) \rightarrow \mathbb{R}$. The most outstanding difference with the first-order case is that the Legendre and Poincaré-Cartan forms associated with a second-order Lagrangian density are generally defined on $J^{3} E$, thus increasing by one the order of the density.

Similarly to the first-order case (see $[11,24]$ ), given a second-order Lagrangian density $\Lambda$ on $p: E \rightarrow N$ and a second-order connection $\gamma^{2}$ on $p: E \rightarrow N$, by subtracting $\left(p_{2}^{3}\right)^{*} \theta^{2}$ from $\left(p_{1}^{3}\right)^{*} \gamma^{2}$ we obtain a $p^{3}$-horizontal form, and we can define the corresponding covariant Hamiltonian to be the Lagrangian density $\Lambda^{\gamma^{2}}$ of third order,

$$
\Lambda^{\gamma^{2}}=\left(\left(p_{1}^{3}\right)^{*} \gamma^{2}-\left(p_{2}^{3}\right)^{*} \theta^{2}\right) \wedge \omega_{\Lambda}-\Lambda
$$

Expanding on the right-hand side of the previous equation, we obtain a decomposition of $\Theta_{\Lambda}$ that generalizes the classical formula for the Hamiltonian in Mechanics; namely, $\Theta_{\Lambda}=\left(p_{1}^{3}\right)^{*} \gamma^{2} \wedge \omega_{\Lambda}-\Lambda^{\gamma^{2}}$. With the same notations as in the formulas (4.1), (4.7), and (4.8) the following formula is deduced:

$$
L^{\gamma^{2}}=\left(\gamma_{i}^{\alpha}+y_{i}^{\alpha}\right) L_{\alpha}^{i 0}+\left(\gamma_{h i}^{\alpha}+y_{h i}^{\alpha}\right) L_{\alpha}^{i h}-L
$$

Because of equation (4.8), $\Theta_{\Lambda}$ and $L^{\gamma^{2}}$ are generally defined on $J^{3} E$.

\subsection{Invariant covariant Hamiltonians on $J^{2} M$}

Lemma 4.2. If $\gamma$ is a first-order Ehresmann connection on $M \times{ }_{N} C^{\mathrm{sym}}$ satisfying the conditions $\left(C_{M}\right)$, then the following equation holds for the second-order Ehresmann connection $\gamma^{2}$ on $M$ given in the formula (4.2):

$$
\gamma_{a b r} \circ \zeta_{N}=-y_{a b, r} .
$$

Proof. Actually, from the formulas (3.8) and (4.3) we obtain

$$
\begin{aligned}
\gamma_{a b r} \circ \zeta_{N}= & -\left(y_{m b}\left(A_{r a}^{m} \circ \zeta_{N}\right)+y_{m a}\left(A_{r b}^{m} \circ \zeta_{N}\right)\right) \\
= & -\frac{1}{2}\left\{y_{m b} y^{m k}\left(y_{r k, a}+y_{a k, r}-y_{r a, k}\right)\right. \\
& \left.+y_{m a} y^{m k}\left(y_{r k, b}+y_{b k, r}-y_{r b, k}\right)\right\} \\
= & -y_{a b, r} .
\end{aligned}
$$


Lemma 4.3. If a first-order connection $\gamma$ on $M \times_{N} C^{\mathrm{sym}}$ satisfies the condition $\left(C_{C}\right)$ introduced above, then the following formulas for its components hold:

$$
\gamma_{r t s}^{h}-\gamma_{r s t}^{h}=A_{s m}^{h} A_{r t}^{m}-A_{t m}^{h} A_{r s}^{m}
$$

Proof. As the bundle under consideration is that of symmetric connections, the following symmetry holds: $\gamma_{a b c}^{h}=\gamma_{b a c}^{h}$, and we have

$$
\begin{aligned}
\gamma_{r t s}^{h}= & \gamma_{s t r}^{h}-\left(A_{r m}^{h} A_{s t}^{m}-A_{s m}^{h} A_{r t}^{m}\right) \quad \text { [by virtue of (3.13)] } \\
= & \gamma_{t s r}^{h}-\left(A_{r m}^{h} A_{s t}^{m}-A_{s m}^{h} A_{r t}^{m}\right) \\
= & \left(\gamma_{r s t}^{h}+A_{r m}^{h} A_{s t}^{m}-A_{t m}^{h} A_{r s}^{m}\right) \quad \text { [by virtue of (3.13)] } \\
& -\left(A_{r m}^{h} A_{s t}^{m}-A_{s m}^{h} A_{r t}^{m}\right) \\
= & \gamma_{r s t}^{h}+\left(A_{s m}^{h} A_{r t}^{m}-A_{t m}^{h} A_{r s}^{m}\right) .
\end{aligned}
$$

Proposition 4.4. Let

$$
\zeta_{N}^{2}=\left.\zeta_{N}^{(1)}\right|_{J^{2} M}: J^{2} M \rightarrow J^{1}\left(M \times{ }_{N} C^{\mathrm{sym}}\right)
$$

be the restriction to the closed submanifold $J^{2} M \subset J^{1}\left(J^{1} M\right)$ of the prolongation $\zeta_{N}^{(1)}: J^{1}\left(J^{1} M\right) \rightarrow J^{1}\left(M \times{ }_{N} C^{\mathrm{sym}}\right)$ of the mapping $\zeta_{N}$ defined in Theorem 4.1. For every $\left(j_{x}^{1} g, j_{x}^{1} \Gamma\right) \in J^{1}\left(M \times{ }_{N} C^{\mathrm{sym}}\right)$ there exists a unique $j_{x}^{2} g^{\prime} \in$ $J_{x}^{2} M$ such that, $j_{x}^{1} g^{\prime}=j_{x}^{1} g$ and $j_{x}^{1} \Gamma^{g^{\prime}}=j_{x}^{1} \Gamma$ and the mapping $\varkappa: J^{1}\left(M \times_{N}\right.$ $\left.C^{\text {sym }}\right) \rightarrow J^{2} M$ defined by $\varkappa\left(j_{x}^{1} g, j_{x}^{1} \Gamma\right)=j_{x}^{2} g^{\prime}$ is a Diff $N$-equivariant rectract of $\zeta_{N}^{2}$.

Proof. From the formulas (4.3) and (4.4) we obtain

$$
\begin{aligned}
\frac{\partial g_{i j}^{\prime}}{\partial x^{k}} & =g_{h i}^{\prime}\left(\Gamma^{g^{\prime}}\right)_{j k}^{h}+g_{h j}^{\prime}\left(\Gamma^{g^{\prime}}\right)_{i k}^{h}, \\
\left(\Gamma^{g^{\prime}}\right)_{i j}^{h} & =\frac{1}{2} g^{\prime h k}\left(\frac{\partial g_{i k}^{\prime}}{\partial x^{j}}+\frac{\partial g_{j k}^{\prime}}{\partial x^{i}}-\frac{\partial g_{i j}^{\prime}}{\partial x^{k}}\right)
\end{aligned}
$$


for every non-singular metric $g^{\prime}$ on $N$. Hence the second partial derivatives of $g_{i j}^{\prime}$ are completely determined, namely

$$
\frac{\partial^{2} g_{i j}^{\prime}}{\partial x^{k} \partial x^{l}}=\frac{\partial g_{h i}}{\partial x^{l}} \Gamma_{j k}^{h}+g_{h i} \frac{\partial \Gamma_{j k}^{h}}{\partial x^{l}}+\frac{\partial g_{h j}}{\partial x^{l}} \Gamma_{i k}^{h}+g_{h j} \frac{\partial \Gamma_{i k}^{h}}{\partial x^{l}}
$$

Moreover, the Levi-Civita connection of a metric depends functorially on the metric, i.e., $\phi \cdot \Gamma^{g}=\Gamma^{\phi \cdot g}$ for every $\phi \in \operatorname{Diff} N$. Hence, by transforming the equations $j_{x}^{1} g^{\prime}=j_{x}^{1} g$ and $j_{x}^{1} \Gamma^{g^{\prime}}=j_{x}^{1} \Gamma^{g}$ by $\phi$ we can conclude.

Theorem 4.5. If a first-order Ehresmann connection $\gamma$ on $M \times{ }_{N} C^{\mathrm{sym}}$ satisfies the conditions $\left(C_{M}\right)$ and $\left(C_{C}\right)$ introduced above, then the covariant Hamiltonian $\Lambda^{\gamma^{2}}$ attached to every $\operatorname{Diff} N$-invariant second-order Lagrangian density $\Lambda$ on $M$ with respect to the second-order Ehresmann connection $\gamma^{2}$ on $M$ defined in formula (4.2), is defined on $J^{2} M$ and it is also Diff $N$ invariant.

Proof. Given a Diff $N$-invariant second-order Lagrangian density $\Lambda=\mathcal{L} \mathbf{v}$ on $M$, let $\Lambda^{\prime}=\mathcal{L}^{\prime} \mathbf{v}$ be the first-order Lagrangian density on $M \times_{N} C^{\text {sym }}$ given by $\Lambda^{\prime}=\varkappa^{*} \Lambda$, which is also Diff $N$-invariant as $\varkappa$ is a Diff $N$-equivariant mapping according to Proposition 4.4. Moreover, as $\varkappa$ is a retract of $\zeta_{N}^{2}$, we have $\left(\zeta_{N}^{2}\right)^{*} \Lambda^{\prime}=\left(\zeta_{N}^{2}\right)^{*} \varkappa^{*} \Lambda=\left(\varkappa \circ \zeta_{N}^{2}\right)^{*} \Lambda=\Lambda$, i.e., $\Lambda=\left(\zeta_{N}^{2}\right)^{*} \Lambda^{\prime}$. This formula is equivalent to saying $\mathcal{L}=\mathcal{L}^{\prime} \circ \zeta_{N}^{2}$, as the $n$-form $\mathbf{v}$ is Diff $N$-invariant, and it is even equivalent to $L=L^{\prime} \circ \zeta_{N}^{2}$ because $\zeta_{N}^{2}$ induces the identity on $N$.

We claim $\mathcal{L}^{\gamma^{2}}=\left(\mathcal{L}^{\prime}\right)^{\gamma} \circ \zeta_{N}^{2}$. This formula will end the proof as the mapping $\zeta_{N}^{2}$ is Diff $N$-equivariant and $\left(\mathcal{L}^{\prime}\right)^{\gamma}$ is $\operatorname{Diff} N$-invariant by virtue of Theorem 3.6.

To start with, we observe that formula (4.7) for $\Lambda$ can be written, in the present case, as follows:

$$
L^{a b i j}=\frac{1}{2-\delta_{i j}} \frac{\partial L}{\partial y_{a b, i j}}
$$

or equivalently, letting $\mathcal{L}^{a b i j}=\rho^{-1} L^{a b i j}$,

$$
\mathcal{L}^{a b i j}=\frac{1}{2-\delta_{i j}} \frac{\partial \mathcal{L}}{\partial y_{a b, i j}} .
$$


Taking the formula in Lemma 4.2 into account, formula (4.10) for $\Lambda$ reads as $L^{\gamma^{2}}=\sum_{a \leq b}\left(\gamma_{a b i j}+y_{a b, i j}\right) L^{a b i j}-L$, or even

$$
\mathcal{L}^{\gamma^{2}}=\sum_{a \leq b}\left(\gamma_{a b i j}+y_{a b, i j}\right) \mathcal{L}^{a b i j}-\mathcal{L}
$$

where $\mathcal{L}^{\gamma^{2}}=\rho^{-1} L^{\gamma^{2}}$. Hence $\mathcal{L}^{\gamma^{2}}$ is defined over $J^{2} M$. As $y_{a b, i j}=y_{a b, j i}$, we obtain

$$
\begin{aligned}
\mathcal{L}^{\gamma^{2}}= & \sum_{a \leq b} \sum_{i \leq j}\left(\frac{1}{2}\left(\gamma_{a b i j}+\gamma_{a b j i}\right)+y_{a b, i j}\right) \frac{\partial\left(\mathcal{L}^{\prime} \circ \zeta_{N}^{2}\right)}{\partial y_{a b, i j}}-\mathcal{L}^{\prime} \circ \zeta_{N}^{2} \\
= & \sum_{a \leq b} \sum_{i \leq j} \sum_{k \leq l}\left(\frac{1}{2}\left(\gamma_{a b i j}+\gamma_{a b j i}\right)+y_{a b, i j}\right) \\
& \times\left(\frac{\partial \mathcal{L}^{\prime}}{\left.\partial A_{k l, q}^{h} \circ \zeta_{N}^{2}\right)} \frac{\partial\left(A_{k l, q}^{h} \circ \zeta_{N}^{2}\right)}{\partial y_{a b, i j}}-\mathcal{L}^{\prime} \circ \zeta_{N}^{2}\right. \\
= & \sum_{k \leq l} \frac{1}{4} y^{h m}\left(\gamma_{k m q l}+\gamma_{k m l q}+\gamma_{l m q k}+\gamma_{l m k q}-\gamma_{k l q m}-\gamma_{k l m q}\right) \\
& \times\left(\frac{\partial \mathcal{L}^{\prime}}{\partial A_{k l, q}^{h}} \circ \zeta_{N}^{2}\right)+\sum_{k \leq l} \frac{1}{2} y^{h m}\left(y_{k m, q l}+y_{l m, q k}-y_{k l, q m}\right) \\
& \times\left(\frac{\partial \mathcal{L}^{\prime}}{\partial A_{k l, q}^{h}} \circ \zeta_{N}^{2}\right)-\mathcal{L}^{\prime} \circ \zeta_{N}^{2} .
\end{aligned}
$$

Moreover, we have

$$
\left(\mathcal{L}^{\prime}\right)^{\gamma}=\sum_{a \leq b}\left(\gamma_{a b c}+y_{a b, c}\right) \frac{\partial \mathcal{L}^{\prime}}{\partial y_{a b, c}}+\sum_{a \leq b}\left(\gamma_{a b l}^{i}+A_{a b, l}^{i}\right) \frac{\partial \mathcal{L}^{\prime}}{\partial A_{a b, l}^{i}}-\mathcal{L}^{\prime} .
$$

Hence

$$
\begin{aligned}
\left(\mathcal{L}^{\prime}\right)^{\gamma} \circ \zeta_{N}^{2}= & \sum_{k \leq l}\left(\gamma_{k l q}^{h} \circ \zeta_{N}+A_{k l, q}^{h} \circ \zeta_{N}\right)\left(\frac{\partial \mathcal{L}^{\prime}}{\partial A_{k l, q}^{h}} \circ \zeta_{N}^{2}\right)-\mathcal{L}^{\prime} \circ \zeta_{N}^{2} \\
= & \sum_{k \leq l}\left\{-\frac{1}{2}\left(\gamma_{k l r q}-\gamma_{l r k q}-\gamma_{r k l q}\right) y^{r h}\right. \\
& \left.+\frac{1}{2}\left(y_{k r, l q}+y_{l r, k q}-y_{k l, r q}\right) y^{h r}\right\}\left(\frac{\partial \mathcal{L}^{\prime}}{\partial A_{k l, q}^{h}} \circ \zeta_{N}^{2}\right)-\mathcal{L}^{\prime} \circ \zeta_{N}^{2}
\end{aligned}
$$


Consequently, the proof reduces to state that the following equation:

$$
\frac{1}{4}\left(\gamma_{k r q l}+\gamma_{k r l q}+\gamma_{l r q k}+\gamma_{l r k q}-\gamma_{k l q r}-\gamma_{k l r q}\right)=-\frac{1}{2}\left(\gamma_{k l r q}-\gamma_{l r k q}-\gamma_{r k l q}\right)
$$

holds true, or equivalently,

$$
0=\left(\gamma_{i j k r}-\gamma_{i j r k}\right)+\left(\gamma_{i r j k}-\gamma_{i r k j}\right)+\left(\gamma_{r j k i}-\gamma_{r j i k}\right)
$$

According to formulas (4.5) and (3.8), we obtain

$$
\begin{aligned}
\gamma_{i j k r} \circ \zeta_{N}^{-1}= & \left(\gamma_{j k r}^{h}-A_{r a}^{h} A_{j k}^{a}\right) y_{h i}+\left(\gamma_{i k r}^{h}-A_{r a}^{h} A_{i k}^{a}\right) y_{h j} \\
& -\left(A_{r j}^{h} A_{i k}^{a}+A_{r i}^{h} A_{j k}^{a}\right) y_{a h} .
\end{aligned}
$$

The third term on the right-hand side of this equation is symmetric in the indices $k$ and $r$, as $A_{b c}^{a}=A_{c b}^{a}$. Hence,

$$
\begin{aligned}
\left(\gamma_{i j k r}-\gamma_{i j r k}\right) \circ \zeta_{N}^{-1}= & \left(\gamma_{j k r}^{h}-\gamma_{j r k}^{h}-A_{r a}^{h} A_{j k}^{a}+A_{k a}^{h} A_{j r}^{a}\right) y_{h i} \\
& +\left(\gamma_{i k r}^{h}-\gamma_{i r k}^{h}-A_{r a}^{h} A_{i k}^{a}+A_{k a}^{h} A_{i r}^{a}\right) y_{h j}
\end{aligned}
$$

By composing the right-hand side of equation (4.13) and $\zeta_{N}^{-1}$, and taking the previous formula and formulas (3.13) and (4.11) into account, we conclude that this expression vanishes indeed.

\section{$5 \quad$ Palatini and Einstein-Hilbert Lagrangians}

Let us compute the covariant Hamiltonian density attached to the Palatini Lagrangian. Following the notations in [20], the Ricci tensor field attached to the symmetric connection $\Gamma$ is given by $S^{\Gamma}(X, Y)=\operatorname{tr}\left(Z \mapsto R^{\Gamma}(Z, X) Y\right)$, where $R^{\Gamma}$ denotes the curvature tensor field of the covariant derivative $\nabla^{\Gamma}$ associated to $\Gamma$ on the tangent bundle; hence $S^{\Gamma}=\left(R^{\Gamma}\right)_{j l} d x^{l} \otimes d x^{j}$, where

$$
\begin{aligned}
\left(R^{\Gamma}\right)_{j l} & =\left(R^{\Gamma}\right)_{j k l}^{k}, \\
\left(R^{\Gamma}\right)_{j k l}^{i} & =\partial \Gamma_{j l}^{i} / \partial x^{k}-\partial \Gamma_{j k}^{i} / \partial x^{l}+\Gamma_{j l}^{m} \Gamma_{k m}^{i}-\Gamma_{j k}^{m} \Gamma_{l m}^{i} .
\end{aligned}
$$

The Lagrangian is the function on $J^{1}\left(M \times{ }_{N} C^{\text {sym }}\right)$ thus given by,

$$
\mathcal{L}_{P}\left(j_{x}^{1} g, j_{x}^{1} \Gamma\right)=g^{i j}(x)\left(R^{\Gamma}\right)_{i j}(x)
$$


and local expression

$$
\mathcal{L}_{P}=y^{i j}\left(A_{i j, k}^{k}-A_{i k, j}^{k}+A_{i j}^{m} A_{k m}^{k}-A_{i k}^{m} A_{j m}^{k}\right) .
$$

As a computation shows, for every first-order connection $\gamma$ on $M \times{ }_{N} C^{\text {sym }}$ satisfying (4.11) and taking the formula (1.2) into account, we obtain $\mathcal{L}_{P}^{\gamma}=$ 0 . This result is essentially due to the fact that the $\mathrm{P}-\mathrm{C}$ form of the $\mathrm{P}$ density $\Lambda_{P}=\mathcal{L}_{P} \mathbf{v}=L_{P} v_{n}$ projects onto $M \times{ }_{N} C^{\text {sym }}$. In fact, the following general characterization holds:

Proposition 5.1. Let $p: E \rightarrow N$ be an arbitrary fibred manifold and let $\gamma$ be a first-order Ehresmann connection on E. The equation $L^{\gamma}=0$ holds true for a Lagrangian $L \in C^{\infty}\left(J^{1} E\right)$ if and only if, (i) the Poincaré-Cartan form of the density $\Lambda=L v_{n}$ projects onto $J^{0} E$ and, (ii) $L=\left\langle\left(p_{0}^{1}\right)^{*} \gamma-\right.$ $\left.\theta,\left.d L\right|_{V\left(p_{0}^{1}\right)}\right\rangle$.

Proof. The equation $L^{\gamma}=0$ is equivalent to the equation $D^{\gamma} L=L$, where $D^{\gamma}$ is the $p_{0}^{1}$-vertical vector field defined in the formula (3.2), and the general solution to the latter is $L=f\left(x^{i}, y^{\alpha}, \gamma_{i}^{\alpha}+y_{i}^{\alpha}\right), f\left(x^{i}, y^{\alpha}, y_{i}^{\alpha}\right)$ being a homogeneous smooth function of degree one in the variables $\left(y_{i}^{\alpha}\right), 1 \leq \alpha \leq m$, $1 \leq i \leq n$, according to Euler's homogeneous function theorem. As $f$ is defined for all values of the variables $\left(y_{i}^{\alpha}\right), 1 \leq \alpha \leq m, 1 \leq i \leq n$, we conclude that the functions $L_{\alpha}^{i}=\partial L / \partial y_{i}^{\alpha}$ must be defined on $E$. Hence $L$ is written as $L=L_{\alpha}^{i}\left(x^{j}, y^{\beta}\right) y_{i}^{\alpha}+L_{0}\left(x^{j}, y^{\beta}\right)$, but this is exactly the condition for the P-C form of $\Lambda$ to be projectable onto $J^{0} E=E$, as follows from the local expression of this form, namely,

$$
\begin{aligned}
\Theta_{\Lambda} & =\frac{\partial L}{\partial y_{i}^{\alpha}} \theta^{\alpha} \wedge i_{\partial / \partial x^{i}} v_{n}+L v_{n} \\
& =\frac{\partial L}{\partial y_{i}^{\alpha}} d y^{\alpha} \wedge i_{\partial / \partial x^{i}} v_{n}+\left(L-y_{i}^{\alpha} \frac{\partial L}{\partial y_{i}^{\alpha}}\right) v_{n}
\end{aligned}
$$

Moreover, by imposing the condition $D^{\gamma} L=L$ we obtain $L_{0}=L_{\alpha}^{i} \gamma_{i}^{\alpha}$, or in other words $L=\left(\gamma_{i}^{\alpha}+y_{i}^{\alpha}\right) \partial L / \partial y_{i}^{\alpha}$, which is equivalent to equation (ii) in the statement.

The corresponding result for the second-order formalism is similar but the computations are more cumbersome. Let us compute the covariant Hamiltonian density attached to the Einstein-Hilbert Lagrangian. As a matter of notation, we set $S^{g}(X, Y)=S^{\Gamma^{g}}(X, Y)$ for the metric $g, \Gamma^{g}$ being its Levi-Civita connection, and similarly, $\left(R^{g}\right)_{j k l}^{i}=\left(R^{\Gamma^{g}}\right)_{j k l}^{i}$. 
The E-H Lagrangian is thus given by $\mathcal{L}_{\mathrm{EH}} \circ j^{2} g=\left(y^{i j} \circ g\right)\left(R^{g}\right)_{i h j}^{h}$. As the Levi-Civita connection $\Gamma^{g}$ depends functorially on $g, \mathcal{L}_{\mathrm{EH}}$ is readily seen to be Diff $N$-invariant; it is in addition linear in the second-order variables $y_{i j, k l}$. By using the third formula in (4.3) the following local expression for $\mathcal{L}_{\mathrm{EH}}$ is obtained:

$$
\begin{aligned}
\mathcal{L}_{\mathrm{EH}}= & \frac{1}{2} y^{i j} y^{h d}\left(y_{d j, h i}-y_{i j, d h}-y_{d h, i j}+y_{h i, d j}\right)+\mathcal{L}_{\mathrm{EH}}^{\prime}, \\
\mathcal{L}_{\mathrm{EH}}^{\prime}= & \frac{1}{2} y^{i j}\left\{y^{h m} y_{m r, j} y^{r d}\left(y_{i d, h}+y_{h d, i}-y_{i h, d}\right)\right. \\
& -y^{h m} y_{m r, h} y^{r d}\left(y_{i d, j}+y_{j d, i}-y_{i j, d}\right) \\
& +\frac{1}{2} y^{h r} y^{m d}\left(y_{i d, j}+y_{j d, i}-y_{i j, d}\right)\left(y_{h r, m}+y_{m r, h}-y_{h m, r}\right) \\
& \left.-\frac{1}{2} y^{h r} y^{m d}\left(y_{i d, h}+y_{h d, i}-y_{i h, d}\right)\left(y_{j r, m}+y_{m r, j}-y_{j m, r}\right)\right\} .
\end{aligned}
$$

According to (4.12), for every first-order connection form $\gamma$ on $M \times{ }_{N} C^{\text {sym }}$ satisfying the conditions $\left(C_{M}\right)$ and $\left(C_{C}\right)$ above, we have

$$
\mathcal{L}_{\mathrm{EH}}^{\gamma^{2}}=\sum_{a \leq b} \frac{1}{2-\delta_{i j}}\left(\gamma_{a b i j}+y_{a b, i j}\right) \frac{\partial \mathcal{L}_{\mathrm{EH}}}{\partial y_{a b, i j}}-\mathcal{L}_{\mathrm{EH}},
$$

and as a computation shows,

$$
\begin{aligned}
\mathcal{L}_{\mathrm{EH}}^{\gamma^{2}}= & \frac{1}{2} y^{i j}\left(\gamma_{i d j h}+\gamma_{j d i h}-\gamma_{i j d h}-\gamma_{i d h j}-\gamma_{h d i j}+\gamma_{i h d j}\right) y^{h d} \\
& +\frac{1}{2} y^{i j}\left\{y^{h m} y_{m r, h} y^{r d}\left(y_{i d, j}+y_{j d, i}-y_{i j, d}\right)\right. \\
& -y^{h m} y_{m r, j} y^{r d}\left(y_{i d, h}+y_{h d, i}-y_{i h, d}\right) \\
& -\frac{1}{2} y^{h r} y^{m d}\left(y_{i d, j}+y_{j d, i}-y_{i j, d}\right)\left(y_{h r, m}+y_{m r, h}-y_{h m, r}\right) \\
& \left.+\frac{1}{2} y^{h r} y^{m d}\left(y_{i d, h}+y_{h d, i}-y_{i h, d}\right)\left(y_{j r, m}+y_{m r, j}-y_{j m, r}\right)\right\} \\
= & 0,
\end{aligned}
$$

where the formulas (4.6), (4.11), (4.3), and Lemma 4.3 have been used. In this case, the $\mathrm{P}-\mathrm{C}$ form of the $\mathrm{E}-\mathrm{H}$ density $\Lambda_{\mathrm{EH}}=\mathcal{L}_{\mathrm{EH}} \mathbf{v}=L_{\mathrm{EH}} v_{n}$,

$$
\begin{aligned}
\Theta_{\Lambda_{\mathrm{EH}}} & =\sum_{k \leq l}\left(L_{\mathrm{EH}}^{i, k l} d y_{k l}+L_{\mathrm{EH}}^{i j, k l} d y_{k l, j}\right) \wedge i_{\partial / \partial x^{i}} v_{n}+H v_{n}, \\
H & =L_{\mathrm{EH}}^{\prime}-\sum_{k \leq l} L_{\mathrm{EH}}^{i, k l} y_{k l, i},
\end{aligned}
$$




$$
\begin{aligned}
L_{\mathrm{EH}}^{i, k l} & =\frac{\partial L_{\mathrm{EH}}^{\prime}}{\partial y_{k l, i}}-\frac{1}{2-\delta_{i j}} y_{a b, j} \frac{\partial^{2} L_{\mathrm{EH}}}{\partial y_{a b} \partial y_{k l, i j}}, \\
L_{\mathrm{EH}}^{i j, k l} & =\frac{1}{2-\delta_{i j}} \frac{\partial L_{\mathrm{EH}}}{\partial y_{k l, i j}},
\end{aligned}
$$

(cf. (4.7), (4.8)) is not only projectable onto $J^{2} M$ but also on $J^{1} M$ (e.g., see [13]), although there is no first-order Lagrangian on $J^{1} M$ admitting (5.1) as its P-C form. This fact is strongly related to a classical result by Hermann Weyl ( [39, Appendix II], also see $[18,22]$ ) according to which the only Diff $N$-invariant Lagrangians on $J^{2} M$ depending linearly on the second-order coordinates $y_{a b, i j}$ are of the form $\lambda \mathcal{L}_{\mathrm{EH}}+\mu$, for scalars $\lambda, \mu$. This also explains why a true first-order Hamiltonian formalism exists in the Einstein-Cartan gravitation theory, e.g., see $[37,38]$. In fact, if

$$
L_{\mathrm{EH}}^{i}=\frac{1}{2-\delta_{i j}} \frac{\partial L_{\mathrm{EH}}}{\partial y_{k l, i j}} y_{k l, j} \quad\left(\text { hence } L_{\mathrm{EH}}^{i j, k l}=\frac{\partial L_{\mathrm{EH}}^{i}}{\partial y_{k l, j}}\right)
$$

and the momentum functions are defined as follows:

$$
p_{k l, i}=L_{\mathrm{EH}}^{i, k l}-\frac{\partial L_{\mathrm{EH}}^{i}}{\partial y_{k l}},
$$

then

$$
d \Theta_{\Lambda_{\mathrm{EH}}}=d p_{k l, i} \wedge d y_{k l} \wedge i_{\partial / \partial x^{i}} v_{n}+d H \wedge v_{n},
$$

and from the Hamilton-Cartan equation (e.g., see [13, (1)]) we conclude that a metric $g$ is an extremal for $\Lambda_{\mathrm{EH}}$ if and only if,

$$
\begin{aligned}
& 0=\frac{\partial\left(p_{a b, i} \circ j^{1} g\right)}{\partial x^{i}}-\frac{\partial H}{\partial y_{a b}} \circ j^{1} g, \\
& 0=\frac{\partial\left(y_{a b} \circ g\right)}{\partial x^{i}}+\frac{\partial H}{\partial y_{a b, i}} \circ j^{1} g .
\end{aligned}
$$

On the other hand, it is no longer true that the covariant Hamiltonians of the non-linear Lagrangians of the form $f\left(\mathcal{L}_{\mathrm{EH}}\right), f^{\prime \prime} \neq 0$, considered in some cosmological models (e.g., see $[1,6,9,12,19,21,31])$ and those in higher dimensions (e.g., see $[15,36]$ ) vanish. In fact, as a computation shows, one has $f\left(\mathcal{L}_{\mathrm{EH}}\right)^{\gamma^{2}}=f^{\prime}\left(\mathcal{L}_{\mathrm{EH}}\right) \mathcal{L}_{\mathrm{EH}}-f\left(\mathcal{L}_{\mathrm{EH}}\right), \forall f \in C^{\infty}(\mathbb{R})$. 
"ATMP-16-3-A3-MUN" — 2013/1/31 — 12:20 — page 884 — \#34

\section{Acknowledgment}

Supported by Ministerio de Ciencia e Innovación of Spain, under grant no. MTM2011-22528.

\section{References}

[1] A. Borowiec, M. Ferraris, M. Francaviglia and I. Volovich, Almostcomplex and almost-product Einstein manifolds from a variational principle, J. Math. Phys. 40(7) (1999), 3446-3464.

[2] U. Bruzzo, The global Utiyama theorem in Einstein-Cartan theory, J. Math. Phys. 28(9) (1987), 2074-2077.

[3] H. Burton and R.B. Mann, Palatini variational principle for an extended Einstein-Hilbert action, Phys. Rev. D (3) 57(8) (1998), $4754-4759$.

[4] - Palatini variational principle for $N$-dimensional dilaton gravity, Classical Quantum Gravity 15(5) (1998), 1375-1385.

[5] M. Castrillón López and J. Muñoz Masqué, The geometry of the bundle of connections, Math. Z. 236 (2001), 797-811.

[6] S. Cotsakis, J. Miritzis and L. Querella, Variational and conformal structure of nonlinear metric-connection gravitational Lagrangians, J. Math. Phys. 40(6) (1999), 3063-3071.

[7] M. Crampin, E. Martínez and W. Sarlet, Linear connections for systems of second-order ordinary differential equations, Ann. Inst. H. Poincaré, Section A, 65(2) (1996), 223-249.

[8] A. De Paris and A. Vinogradov, Fat manifolds and linear connections, World Scientific Publishing Co. Pte. Ltd., Hackensack, NJ, 2009.

[9] J.P. Duruisseau and R. Kerner, The effective gravitational Lagrangian and the energy-momentum tensor in the inflationary universe, Classical Quantum Gravity 3(5) (1986), 817-824.

[10] F. Etayo Gordejuela and J. Muñoz Masqué, Gauge group and G-structures, J. Phys. A: Math. Gen. 28(2) (1995), 497-510.

[11] A. Fernández, P.L. García and J. Muñoz Masqué, Gaugeinvariant covariant Hamiltonians, J. Math. Phys. 41 (2000), 52925303.

[12] É.É. Flanagan, Palatini form of $1 / R$ gravity, Phys. Rev. Lett. 92(7) (2004), 071101, 4 pp.

[13] P.L. García and J. Muñoz Masqué, Le problème de la régularité dans le calcul des variations du second ordre, C.R. Acad. Sci. Paris Série I 301 (1985), 639-642. 
[14] - Differential invariants on the bundles of linear frames, J. Geom. Phys. 7(3) (1990), 395-418.

[15] B. Giorgini and R. Kerner, Cosmology in ten dimensions with the generalised gravitational Lagrangian, Classical Quantum Gravity 5(2) (1988), 339-351.

[16] H. Goldschimdt and S. Sternberg, The Hamilton-Cartan formalism in the calculus of variations, Ann. Inst. Fourier, Grenoble 23 (1973), 203-267.

[17] M.J. Gotay, An Exterior Differential Systems Approach to the Cartan form, Symplectic Geometry and Mathematical Physics, eds. P. Donato, C. Duval, J. Elhadad and G.M. Tuynman, Boston, Birkhäuser, 1991, 160-188.

[18] P. Von der Heyde, A generalized Lovelock theorem for the gravitational field with torsion, Phys. Lett. A (3) 51 (1975), 381-382.

[19] R. Kerner, Cosmology without singularity and nonlinear gravitational Lagrangians, Gen. Relativity Gravitation 14(5) (1982), 453-469.

[20] S. Kobayashi and K. Nomizu, Foundations of differential Geometry, Volume I, John Wiley \& Sons, Inc. NY, 1963.

[21] T. Koivisto and H. Kurki-Suonio, Cosmological perturbations in the Palatini formulation of modified gravity, Classical Quantum Gravity 23(7) (2006), 2355-2369.

[22] D. Lovelock, The einstein tensor and its generalizations, J. Math. Phys. 12 (1971), 498-501.

[23] L. Mangiarotti and G. Sardanashvily, Connections in Classical and Quantum Field Theory, World Scientific Publishing Co. Inc. River Edge, NJ, 2000.

[24] J. Marsden and S. Shkoller, Multisymplectic geometry, covariant Hamiltonians, and water waves, Math. Proc. Cambridge Phil. Soc. 125 (1999), 553-575.

[25] E. Massa and E. Pagani, Jet bundle geometry, dynamical connections, and the inverse problem of Lagrangian mechanics, Ann. Inst. H. Poincaré Phys. Théor. 61(1) (1994), 17-62.

[26] J. Muñoz Masqué, An axiomatic characterization of the PoincaréCartan form for second-order variational problems, Lecture Notes in Math. 1139, Springer-Verlag 1985, 74-84.

[27] J. Muñoz Masqué and L.M. Pozo Coronado, Parameter Invariance in field theory and the Hamiltonian formalism, Fortschr. Phys. 48(4) (2000), 361-405.

[28] J. Muñoz Masqué and M. Eugenia Rosado, Invariant variational problems on linear frame bundles, J. Phys. A: Math. Gen. 35 (2002), 2013-2036. 
"ATMP-16-3-A3-MUN" — 2013/1/31 — 12:20 — page 886 — \#36

[29] — The problem of invariance for covariant Hamiltonians, Rend. Sem. Mat. Univ. Padova 120 (2008), 1-28.

[30] J. Muñoz Masqué and A. Valdés Morales, The number of functionally independent invariants of a pseudo-Riemannian metric, J. Phys. A: Math. Gen. 27 (1994), 7843-7855.

[31] N. Popławski, The cosmic snap parameter in $f(R)$ gravity, Classical Quantum Gravity 24(11) (2007), 3013-3020.

[32] G.A. Sardanashvily, Gauge theory in jet manifolds, Hadronic Press Monographs in Applied Mathematics, Hadronic Press, Inc., Palm Harbor, FL, USA, 1993.

[33] G. Sardanashvily and O. Zakharov, Gauge gravitation theory, World Scientific Publishing Co. Inc. River Edge, NJ, 1992.

[34] D.J. Saunders, The geometry of jet bundles, Cambridge University Press, Cambridge, UK, 1989.

[35] D.J. Saunders and M. Crampin, On the Legendre map in higher-order field theories, J. Phys. A: Math. Gen. 23 (1990), 3169-3182.

[36] B. Shahid-Saless, Palatini variation of curvature-squared action and gravitational collapse, J. Math. Phys. 32(3) (1991), 694-697.

[37] W. Szczyrba, The canonical variables, the symplectic structure and the initial value formulation of the generalized Einstein-Cartan theory of gravity, Comm. Math. Phys. 60(3) (1978), 215-232.

[38] — Field equations and contracted Bianchi identities in the generalized Einstein-Cartan theory, Lett. Math. Phys. 2(4) (1977/78), 265-274.

[39] H. Weyl, Space-time-matter, translated by H.L. Brose, Dover Publications, Inc., 1952. 